\title{
DOUBLY REFLECTED BSDES WITH CALL PROTECTION AND THEIR APPROXIMATION
}

\author{
Jean-François Chassagneux ${ }^{1,3}$ and Stéphane Crépey ${ }^{2,3}$
}

\begin{abstract}
We study the numerical approximation of doubly reflected backward stochastic differential equations with intermittent upper barrier (RIBSDEs). These denote reflected BSDEs in which the upper barrier is only active on certain random time intervals. From the point of view of financial interpretation, RIBSDEs arise as pricing equations of game options with constrained callability. In a Markovian set-up we prove a convergence rate for a time-discretization scheme by simulation to an RIBSDE. We also characterize the solution of an RIBSDE as the largest viscosity subsolution of a related system of variational inequalities, and we establish the convergence of a deterministic numerical scheme for that problem. Due to the potentially very high dimension of the system of variational inequalities, this approach is not always practical. We thus subsequently prove a convergence rate for a time-discretisation scheme by simulation to an RIBSDE.
\end{abstract}

Mathematics Subject Classification. 93E20, 65C99, 60H30.

Received April 10, 2012. Revised January 24, 2013.

\section{INTRODUCTION}

In this work, we consider the issue of numerical solution of a doubly reflected backward stochastic differential equation, with an upper barrier which is only active on random time intervals (doubly reflected BSDE with an intermittent upper barrier, or RIBSDE for short henceforth, where the "I" in RIBSDE stands for "intermittent").

From the mathematical point of view, such RIBSDEs and, in the Markovian case, the related variational inequality approach, are considered in $[8,13,16,18,19,22]$. They are a natural extension of reflected BSDEs with one or two barriers $[11,13]$. From the point of view of financial interpretation, RIBSDEs arise as pricing equations of game options (like convertible bonds) with call protection, in which the call times of the option's issuer are subject to constraints preventing the issuer from calling the bond on certain random time intervals. Because of this interpretation, we shall call pricing function the value function of a Dynkin game related to an RIBSDE. In the standing example of convertible bonds, the call protection is typically monitored at discrete times in a possibly very path-dependent way: calls may be allowed or not at a given time depending on past values of the underlying stock $S$. This leads, after extension of the state space to markovianize the problem,

Keywords and phrases. Reflected BSDEs, Variational inequalities, Discrete-time approximation, Game option, Call protection.

1 Department of Mathematics, Imperial College London, SW7A2Z, London, UK. j.chassagneux@imperial.ac.uk

2 L.A.P. Université d'Evry Val d'Essonne, 91037 Evry, France. stephane.crepey@univ-evry.fr

3 This research benefited from the support of the "Chair Markets in Transition" under the aegis of Louis Bachelier laboratory, a joint initiative of École polytechnique, Université d'Évry Val d'Essonne and Fédération Bancaire Française. The paper was completed during a visit to the Hausdorff Research Institute for Mathematics at the University of Bonn in the framework of the Trimester Program Stochastic Dynamics in Economics and Finance. 
TABLE 1. MC versus VI computation times and relative error (NA $\leftrightarrow$ Non Available).

\begin{tabular}{lccccc}
\hline$d$ & 1 & 5 & 10 & 20 & 30 \\
\hline CPU MC & $0.5 \mathrm{~s}$ & $0.6 \mathrm{~s}$ & $0.9 \mathrm{~s}$ & $1.4 \mathrm{~s}$ & $1.9 \mathrm{~s}$ \\
CPU VI & $1.0 \mathrm{~s}$ & $16.1 \mathrm{~s}$ & $465.0 \mathrm{~s}$ & $\mathrm{NA}$ & $\mathrm{NA}$ \\
\hline Rel Err & $0.02 \%$ & $0.02 \%$ & $0.01 \%$ & $0.02 \%$ & $0.02 \%$ \\
\hline
\end{tabular}

to highly-dimensional pricing problems. This was actually our initial motivation for this work, as a financial engineering software company expressed concern about the ability to deal with such kind of clauses with a partial differential equations technology.

Pursuing the study initiated in Section 14.2 of [8], we first prove in a Markov setup an analytical characterization of the pricing function, as the largest viscosity subsolution to a related system of obstacles problems. This proof uses a stability property for the random time intervals related to the call protection, which is taken as an assumption in the slightly more general set-up of [8]. This stability property is established in the context of the present paper in Lemma 5.1. The analytical characterization of the value function allows us then to propose a deterministic scheme, which is shown to be convergent, to solve numerically the RIBSDE (or, more precisely, the corresponding system of variational inequalities), see Proposition 3.9.

But, due to the path dependence of the call protection, deterministic pricing schemes are ruled out by the curse of dimensionality, and simulation methods appear to be the only viable alternative. We thus present a discretetime approximation scheme for an RIBSDE, inspired by [3,6], but taking into account the fact that the upper boundary is only active on some random time intervals. One important step in the proof of convergence for the scheme is to prove the convergence of the approximated random time intervals, which is done in Proposition 4.1.

It should be pointed out that the "irregularity", in some sense, of the upper boundary, implies, in general, a discontinuous value process $Y$. Nevertheless, we are able to retrieve a bound for the convergence rate of the scheme for the $Y$-component, see Theorem 4.3, and a convergence result for the $Z$-component, see Theorem 4.4, in the case where the driver of the RIBSDE does not depend on $Z$. The study of the case where the driver of the RIBSDE depends on $Z$, or the study of the rate of approximation of the $Z$-component, leads to the study of the regularity of the $Z$-component, which is made difficult here by the discontinuity of the $Y$-component.

The practical value of the numerical schemes is thoroughly assessed in the companion paper [10]. For motivation, see Table 1, which gives computation times of the simulation scheme (MC) and of the alternative deterministic numerical scheme for solving the related variational inequalities (VI), for problems of increasing dimension $d$ (dimension $d$ in the context and in the sense of Example 2.8(ii), corresponding to systems of $2^{d}$ variational inequalities).

The relative errors in the last row are computed in reference to a price obtained by a low-dimensional deterministic numerical scheme which is available in the special case under consideration, see [10]. But in the general case only two algorithms are available, (MC) and (VI), and as visible in Table 1, (VI) becomes unpractical for $d$ greater than say 10, whereas computational times and accuracy of (MC) do not seem to be affected by the increasing dimension $d$, at least, not exponentially.

The rest of the paper is organized as follows. Section 2 pursues the study of RIBSDEs initiated in Section 14.2 of [8]. In Section 3, we prove the analytical characterization of the pricing function and study a deterministic pricing scheme. Discrete-time approximation of the RIBSDE is dealt within Section 4. All the proofs are deferred to Section 5, see also the Appendix.

We shall denote:

- $\mathbb{E}_{t} \equiv \mathbb{E}\left(\cdot \mid \mathcal{F}_{t}\right)$,

- $\mathbb{R}^{q}$, the set of $q$-dimensional vectors (or sometimes row-vectors, as should be clear from the context) with real components,

- $|\cdot|_{p}$ for $p \in[1,+\infty)$, or simply $|\cdot|$ in case $p=2$, the $p$-norm of an element of $\mathbb{R}^{q}$,

- $\Lambda$, a positive constant which may change from line to line. 


\subsection{Set-up}

Let be given a continuous time stochastic basis $(\Omega, \mathcal{F}, \mathbb{F}, \mathbb{P})$ over $[0, T]$, where $\mathbb{P}$ would stand for a risk-neutral pricing measure in a financial interpretation. We assume that the filtration $\mathbb{F}$ satisfies the usual completeness and right-continuity conditions, and that all semimartingales are càdlàg ${ }^{4}$. Also, since our practical concern is the pricing of a financial derivative with maturity $T$, we set $\mathbb{F}=\left(\mathcal{F}_{t}\right)_{t \in[0, T]}$ with $\mathcal{F}_{0}$ trivial and $\mathcal{F}_{T}=\mathcal{F}$. A process on $[0, T]$ (respectively a random variable) has to be $\mathbb{F}$-adapted (respectively $\mathcal{F}$-measurable), by definition. By default in the sequel, all "stochastic" identities are to be understood $d \mathbb{P}$ - almost surely or $d \mathbb{P} \otimes d t-$ almost everywhere, respectively.

Given a $q$-dimensional Brownian motion $W(q \geq 1)$, let $X$ be the solution on $[0, T]$ of the following SDE

$$
X_{t}=X_{0}+\int_{0}^{t} b\left(s, X_{s}\right) \mathrm{d} s+\int_{0}^{t} \sigma\left(s, X_{s}\right) \mathrm{d} W_{s}
$$

where the coefficients $b:[0, T] \times \mathbb{R}^{q} \rightarrow \mathbb{R}^{q}$ and $\sigma:[0, T] \times \mathbb{R}^{q} \rightarrow \mathbb{R}^{q \times q}$ are such that:

(Hx) $b, \sigma$ are uniformly bounded by $\Lambda$ and $\Lambda$-Lipschitz continuous in $(t, x)$, i.e.

$$
\left|b(t, x)-b\left(t^{\prime}, x^{\prime}\right)\right|+\left|\sigma(t, x)-\sigma\left(t^{\prime}, x^{\prime}\right)\right| \leq \Lambda\left(\left|t-t^{\prime}\right|+\left|x-x^{\prime}\right|\right),(t, x),\left(t^{\prime}, x^{\prime}\right) \in[0, T] \times \mathbb{R}^{d}
$$

For later use, let us denote by $\mathcal{G}$ the generator of $X$, so for any function $u=u(t, x)$, with $a(t, x)=$ $\sigma(t, x) \sigma(t, x)^{\top}$,

$$
\mathcal{G} u(t, x)=\partial_{t} u(t, x)+\partial_{x} u(t, x) b(t, x)+\frac{1}{2} \operatorname{Tr}\left[a(t, x) \partial_{x^{2}}^{2} u(t, x)\right],
$$

where $\partial_{x} u$ and $\partial_{x^{2}}^{2} u$ denote the row-gradient and the Hessian of a function $u=u(t, x)$ with respect to $x$.

Let be given a set $\mathfrak{T}=\left\{T_{0}, T_{1}, \ldots, T_{N}\right\}$ of fixed times with $0=T_{0}<T_{1}<\ldots<T_{N-1}<T_{N}=T$, and a finite set $\mathcal{K}$. A $\mathcal{K}$-valued argument $k$ of a function $u$ will be equivalently denoted as the last argument $u(\ldots, k)$ or as a superscript $u^{k}(\ldots)$. Thus $k$ can be thought of as referring to the index of a vector or system of functions of the variables other than $k$, if found more convenient in the context at hand.

We suppose that for every $I=0, \ldots, N$, a jump function $\kappa_{I}: \mathbb{R}^{q} \times \mathcal{K} \rightarrow \mathcal{K}$ is given as

$$
\kappa_{I}(x, k)=\kappa_{I,-}^{k} \mathbf{1}_{\{x \in \mathcal{O}\}}+\kappa_{I,+}^{k} \mathbf{1}_{\{x \notin \mathcal{O}\}},
$$

where the $\kappa_{I, \pm}^{k}$ are in $\mathcal{K}$, and where $\mathcal{O}$ is an open domain of $\mathbb{R}^{q}$. We also introduce the algebraic distance function d to $\mathcal{O}$, so $\mathcal{O}=\left\{x \in \mathbb{R}^{q} \mid \mathrm{d}(x)<0\right\}$. The function $x \mapsto \kappa_{I}^{k}(x)$ of this paper is constant in $x$ on $\mathcal{O}$ and outside $\overline{\mathcal{O}}$, and is therefore trivially continuous outside $\partial \mathcal{O}$. In [8], one works with "abstract" functions $\kappa_{I}^{k}$ (denoted there by $\theta_{l}^{i}$, see e.g. the Eq. (14.10) in [8]) and it is frequently assumed that "a certain condition holds at a point $x$ of continuity of $\kappa_{I}^{k}$ ". In view of the above observation and for the sake of simplicity, we shall rather postulate instead, in the context of the present paper, that "a certain condition holds for every $x \notin \partial \mathcal{O}$ ". Moreover, we impose the following regularity and non-characteristic boundary condition on $\mathcal{O}$.

(Ho) The distance function d is of class $\mathcal{C}_{b}^{4}$. Moreover, for every $(t, x)$,

$$
\left(\partial_{x} d a\left(\partial_{x} d\right)^{\top}\right)(t, x) \geq \Lambda^{-1} .
$$

We are now in a position to introduce the factor process $\mathcal{X}=(X, H)$, in which $X$ is defined by (1.1), and a $\mathcal{K}$-valued pure jump marker process $H$ is supposed to be constant except for deterministic jumps at the positive times $T_{I}$, from $H_{T_{I}-}$ to

$$
H_{T_{I}}=\kappa_{I}\left(X_{T_{I}}, H_{T_{I}-}\right)
$$

\footnotetext{
${ }^{4}$ French acronym meaning "right continuous, with left-hand limit".
} 
starting from an initial condition $H_{0}=k \in \mathcal{K}$. In (1.4), $H_{T_{I}-}$ stands for the left-hand limit at $T_{I}$ of the càdlàg process $H$. Note that $H$ does not jump at time $T_{0}=0$.

Let finally be given a non-decreasing sequence of stopping times $\vartheta=\left(\vartheta_{l}\right)_{0 \leq l \leq N+1}$ defined by $\vartheta_{0}=0$ and, for every $l \geq 1$ :

$$
\vartheta_{2 l-1}=\inf \left\{t>\vartheta_{2 l-2} ; H_{t} \notin K\right\} \wedge T, \vartheta_{2 l}=\inf \left\{t>\vartheta_{2 l-1} ; H_{t} \in K\right\} \wedge T,
$$

for a given subset $K$ of $\mathcal{K}$. The $\vartheta_{l}$ are thus $\mathfrak{T}$-valued stopping times, and in particular $\vartheta_{N+1}=T$.

Remark 1.1. The financial interpretation will be developed in Section 2.1. In few words, T represents a set of call protection monitoring times. The marker process $H$ is used for keeping track of the path-dependence of the call protection clauses, in order to make the set-up Markovian. The times $\vartheta_{l}$ s are interpreted as times of switching of call protection.

\section{MARKovian RIBSDE}

We denote by $(P)$ the class of functions $u$ on $\mathbb{R}^{q},[0, T] \times \mathbb{R}^{q}$ or $\mathcal{E}=[0, T] \times \mathbb{R}^{q} \times \mathcal{K}$, such that $u$ is Borelmeasurable, with polynomial growth in its spatial argument $x \in \mathbb{R}^{q}$. Let further be given real-valued and continuous cost functions $g(t, x), \ell(t, x), h(t, x)$ and $f(t, x, y)$ of class $(P)$, for every $y \in \mathbb{R}$ in the case of $f$, such that:

- the running payoff function $f(t, x, y)$ is Lipschitz in $y$;

- the payoff function at maturity $g(x)$ and the put and call payoff functions $\ell(t, x)$ and $h(t, x)$ satisfy $\ell \leq h$, $\ell(T, \cdot) \leq g \leq h(T, \cdot)$.

Remark 2.1. Most developments of this paper can be elevated to the more general case of a $z$-dependent driver $f(t, x, y, z)$. However since one of our main results, Theorem 4.3 , is only for $f=f(t, x, y)$ we restrict ourselves to this case altogether. Note that this restriction is fine with respect to the main real-life application we have in mind, namely the pricing of convertible bonds with call protection. This typically only involves $f=f(t, x, y)$, where the dependence in $y$ corresponds to discounting at some rate in the financial interpretation, see Crépey et al. $[8,10]$.

In the sequel, we shall sometimes use the following assumptions.

$(\mathbf{H} \ell) \ell(t, x)=\lambda(t, x) \vee c$, for a constant $c \in \mathbb{R} \cup\{-\infty\}$ and a function $\lambda$ of class $\mathcal{C}^{1,2}$ on $[0, T] \times \mathbb{R}^{q}$ such that

$$
\lambda, \mathcal{G} \lambda,(\partial \lambda) \sigma \text { are of class }(P),
$$

(Hh) $h(t, x)$ is jointly Lipschitz in $(t, x)$.

The Markovian RIBSDE $(\mathcal{S})$, with data

$$
f\left(t, X_{t}, y\right), g\left(X_{T}\right), \ell\left(t, X_{t}\right), h\left(t, X_{t}\right), \vartheta
$$

is then defined as a doubly reflected BSDE, see e.g., $[8,11]$, with lower and upper barriers respectively given by, for $t \in[0, T]$,

$$
L_{t}=\ell\left(t, X_{t}\right), U_{t}=\sum_{l=0}^{[N / 2]} \mathbf{1}_{t \in\left[\vartheta_{2 l}, \vartheta_{2 l+1}\right)} \infty+\sum_{l=1}^{[(N+1) / 2]} \mathbf{1}_{t \in\left[\vartheta_{2 l-1}, \vartheta_{2 l}\right)} h\left(t, X_{t}\right) .
$$

With respect to standard, "continuously reflected" doubly reflected BSDEs, the peculiarity of RIBSDEs is thus that the "nominal" upper obstacle $h\left(t, X_{t}\right)$ is only active on the "odd" random time intervals $\left[\vartheta_{2 l-1}, \vartheta_{2 l}\right), l>0$. 
Let us introduce the following Banach (or Hilbert, in case of $\mathcal{L}^{2}$ or $\mathcal{H}_{q}^{2}$ ) spaces of random variables or processes, where $p$ denotes here and henceforth a real number in $[1, \infty)$ :

- $\mathcal{L}^{p}$, the space of real valued random variables $\chi$ such that

$$
\|\chi\|_{\mathcal{L}^{p}}=\left(\mathbb{E}\left[|\chi|^{p}\right]\right)^{\frac{1}{p}}<+\infty
$$

- $\mathcal{S}_{q}^{p}$, for any real $p \geq 2$ (or $\mathcal{S}^{p}$, in case $q=1$ ), the space of $\mathbb{R}^{q}$-valued càdlàg processes $Y$ such that

$$
\|Y\|_{\mathcal{S}_{d}^{p}}:=\left(\mathbb{E}\left[\sup _{t \in[0, T]}\left|Y_{t}\right|^{p}\right]\right)^{\frac{1}{p}}<+\infty ;
$$

- $\mathcal{H}_{q}^{p}$ (or $\mathcal{H}^{p}$, in case $q=1$ ), the space of $\mathbb{R}^{q}$-valued predictable processes $Z$ such that

$$
\|Z\|_{\mathcal{H}_{q}^{p}}=\left(\mathbb{E}\left[\left(\int_{0}^{T}\left|Z_{t}\right|^{2} \mathrm{~d} t\right)^{\frac{p}{2}}\right]\right)^{\frac{1}{p}}<+\infty
$$

- $\mathcal{A}^{2}$, the space of finite variation processes $A$ with non-decreasing Jordan components ${ }^{5} A^{ \pm} \in \mathcal{S}^{2}$ null at time 0 .

Note that under $(\mathrm{H} x)$, one has $\|X\|_{\mathcal{S}^{2}} \leq C_{\Lambda}$, where, from now on, $C_{\Lambda}$ stands for a generic constant which depends only on $\Lambda, T, X_{0}, N$ and $q$, and whose value may change from line to line. In case this constant depends on some extra parameter, say $\rho$, we shall write $C_{\Lambda}^{\rho}$.

Definition 2.2. An $(\Omega, \mathbb{F}, \mathbb{P})$-solution $\mathcal{Y}$ to $(\mathcal{S})$ is a triple $\mathcal{Y}=(Y, Z, A)$, such that:

(i) $Y \in \mathcal{S}^{2}, Z \in \mathcal{H}_{q}^{2}, A \in \mathcal{A}^{2}, A^{+}$is continuous; and

$$
\{(\omega, t) ; \Delta Y \neq 0\}=\left\{(\omega, t) ; \Delta A^{-} \neq 0\right\} \subseteq \bigcup_{l=0}^{[N / 2]} \llbracket \vartheta_{2 l} \rrbracket, \Delta Y=\Delta A^{-} \text {on } \bigcup_{l=0}^{[N / 2]} \llbracket \vartheta_{2 l} \rrbracket ;
$$

(ii) $Y_{t}=g\left(X_{T}\right)+\int_{t}^{T} f\left(s, X_{s}, Y_{s}\right) \mathrm{d} s+A_{T}-A_{t}-\int_{t}^{T} Z_{s} \mathrm{~d} W_{s}, t \in[0, T]$;

(iii) $L_{t} \leq Y_{t}, Y_{t} \leq U_{t}, t \in[0, T]$ and $\int_{0}^{T}\left(Y_{t}-L_{t}\right) \mathrm{d} A_{t}^{+}=\int_{0}^{T}\left(U_{t-}-Y_{t-}\right) \mathrm{d} A_{t}^{-}=0$,

where $L$ and $U$ are defined by (2.3), and with the convention that $0 \times+\infty=0$ in (iii).

This definition admits an obvious extension to a random terminal time $\theta$, instead of constant $T$. This extension will be used freely in the sequel, in the special case of simply reflected and continuously doubly reflected BSDEs.

Note that $(\mathcal{S})$ is implicitly parameterized by the initial condition $\left(t=0, X_{0}, k\right)$ of $\mathcal{X}$. We shall use whenever necessary a superscript ${ }^{\xi}$, in reference to an initial condition $\xi=(t, x, k)$ of $\mathcal{X}$. So $\mathcal{X}_{t}^{\xi}=(x, k), \vartheta_{0}^{\xi}=t$. For every initial time $t$, all our processes are extended "in the natural way" to $[0, T]$, see Crépey [8], so that they live in spaces of functions defined over $[0, T]$.

Under $(\mathrm{H} \ell)$, existence and uniqueness of solutions with a continuous reflecting process $A^{l, \xi}$ to the auxiliary reflected BSDEs and doubly reflected BSDEs with random terminal time that appear in point (i) below, is granted by the results of $[8,9]$. Note in particular that under $(\mathrm{H} \ell)$, there exists a quasimartingale $Q=L$ with

\footnotetext{
${ }^{5}$ By the Jordan components of $A$, we mean the terms of the unique decomposition $A=A^{+}-A^{-}$of $A$ as difference of two non-decreasing processes $A^{ \pm}$null at 0 , defining mutually singular random measures on $[0, T]$.
} 
Rao components in $\mathcal{S}^{2}$ such that $L \leq Q \leq U$ over [0,T]. By application of the results of [17], the so-called Mokobodski condition is therefore satisfied by the doubly reflected BSDE problem with the continuously active barriers $\ell\left(t, X_{t}\right)$ and $h\left(t, X_{t}\right)$. This condition is key in the existence issue. As for uniqueness it follows from general a priori error estimates which can be established for RIBSDEs exactly as those of [9] for doubly reflected BSDEs (general estimates in the sense that they do not need a Markov setup).

Proposition 2.3 (Prop. 14.2.11 in [8]; see also [13, 16, 18, 19,22]).

We assume $(\mathrm{H} \ell)$.

(i) The following iterative construction is well-defined, for l decreasing from $N$ to $0: \mathcal{Y}^{l, \xi}=\left(Y^{l, \xi}, Z^{l, \xi}, A^{l, \xi}\right)$ is the unique solution, with $A^{l, \xi}$ continuous, to the reflected BSDE with random terminal time $\vartheta_{l+1}^{\xi}$ (for $l$ even) or the doubly reflected BSDE with random terminal time $\vartheta_{l+1}^{\xi}$ (for l odd) on $\left[t, \vartheta_{l+1}^{\xi}\right]$, with data

$$
\begin{cases}f\left(s, X_{s}^{\xi}, y\right), Y_{\vartheta_{l+1}^{\xi}}^{l+1, \xi}, \ell\left(s, X_{s}^{\xi}\right), \vartheta_{l+1}^{\xi} & \text { (l even) } \\ f\left(s, X_{s}^{\xi}, y\right), \min \left(Y_{\vartheta_{l+1}^{\xi}}^{l+1, \xi}, h\left(\vartheta_{l+1}^{\xi}, X_{\vartheta_{l+1}^{\xi}}^{\xi}\right)\right), \ell\left(s, X_{s}^{\xi}\right), h\left(s, X_{s}^{\xi}\right), \vartheta_{l+1}^{\xi}(l \text { odd })\end{cases}
$$

in which, in case $l=N, Y_{\vartheta_{l+1}^{\xi}}^{l+1, \xi}$ is to be understood as $g\left(X_{T}^{\xi}\right)$.

(ii) Let us define $\mathcal{Y}^{\xi}=\left(Y^{\xi}, Z^{\xi}, A^{\xi}\right)$ on $[t, T]$ by, for every $l=0, \ldots, N$ :

- $\left(Y^{\xi}, Z^{\xi}\right)=\left(Y^{l, \xi}, Z^{l, \xi}\right)$ on $\left[\vartheta_{l}^{\xi}, \vartheta_{l+1}^{\xi}\right)$, and also at $\vartheta_{l+1}^{\xi}=T$ in case $l=N$,

- $d A^{\xi}=d A^{l, \xi}$ on $\left(\vartheta_{l}^{\xi}, \vartheta_{l+1}^{\xi}\right)$, and

$$
\Delta A_{\vartheta_{l}^{\xi}}^{\xi}=Y_{\vartheta_{l}^{\xi}}^{l, \xi}-\min \left(Y_{\vartheta_{l}^{\xi}}^{l, \xi}, h\left(\vartheta_{l}^{\xi}, X_{\vartheta_{l}^{\xi}}^{\xi}\right)\right)=\Delta Y_{\vartheta_{l}^{\xi}}^{\xi}(=0 \text { for } l \text { odd })
$$

and $\Delta A_{T}^{\xi}=\Delta Y_{T}^{\xi}=0$. So in particular (recall that $k$ refers to $\xi=(t, x, k)$ )

$$
Y_{t}^{\xi}= \begin{cases}Y_{t}^{0, \xi} & k \in K \\ Y_{t}^{1, \xi}, & k \notin K\end{cases}
$$

Then $\mathcal{Y}^{\xi}=\left(Y^{\xi}, Z^{\xi}, A^{\xi}\right)$ is the unique solution to the RIBSDE $\left(\mathcal{S}^{\xi}\right)$.

One will need further stability results on the $\mathcal{Y}^{l, \xi}$. Toward this end, a suitable stability assumption on $\vartheta^{\xi}$ is needed. Our next result is essentially a càdlàg property of $\vartheta$, viewed as a random function of the initial condition $\xi=(t, x, k)$ of $\mathcal{X}^{\xi}$. We denote, for $I=1, \ldots, N$,

$$
\mathcal{E}_{I}=\left[T_{I-1}, T_{I}\right] \times \mathbb{R}^{q} \times \mathcal{K}, \quad \mathcal{E}_{I}^{*}=\left[T_{I-1}, T_{I}\right) \times \mathbb{R}^{q} \times \mathcal{K} .
$$

Proposition 2.4. Let $\xi_{n}=\left(t_{n}, x_{n}, k\right) \rightarrow \xi=(t, x, k)$ in $\mathcal{E}$ as $n \rightarrow \infty$.

(i) In case $t \notin \mathfrak{T}$, or in case $t=T_{I}$ and the $\xi_{n}$ are in $\mathcal{E}_{I+1}$, then there exists an extraction $\left(\xi_{n^{\prime}}\right)_{n}$ for which, almost surely, $\vartheta^{\xi_{n^{\prime}}} \rightarrow \vartheta^{\xi}$ as $n \rightarrow \infty$;

(ii) In case $t=T_{I}, x \notin \partial \mathcal{O}$ and the $\xi_{n}$ are in $\mathcal{E}_{I}^{*}$, then there exists an extraction $\left(\xi_{n^{\prime}}\right)_{n}$ for which, almost surely, $\vartheta^{\xi_{n^{\prime}}}$ converges to some non-decreasing sequence $\widetilde{\vartheta}^{\xi}=\left(\widetilde{\vartheta}_{l}^{\xi}\right)_{0 \leq l \leq N+1}$ of $\mathfrak{T}$-valued stopping times as $n \rightarrow \infty$.

Observe that since the $\vartheta_{l}$ are $\mathfrak{T}$-valued stopping times:

- $\vartheta^{\xi_{n^{\prime}}} \rightarrow \vartheta^{\xi}$ in part (i) of the Proposition effectively means that $\vartheta_{l}^{\xi_{n^{\prime}}}=\vartheta_{l}^{\xi}$ for $n^{\prime}$ large enough, almost surely, for every $l=1, \ldots, N+1$;

- the convergence of $\vartheta_{n^{\prime}}$ to $\widetilde{\vartheta}^{\xi}$ in part (ii) of the Proposition effectively means that $\vartheta_{l}^{\xi_{n^{\prime}}}=\widetilde{\vartheta}_{l}^{\xi}$ for $n$ large enough, almost surely, for every $l=1, \ldots, N+1$. 
Definition 2.5. Substituting $\widetilde{\vartheta}^{\xi}$ to $\vartheta^{\xi}$ in the construction of $\mathcal{Y}^{\xi}$ in Proposition 2.3(i), one gets a new sequence of reflected and doubly reflected BSDEs with random terminal times. One denotes by $\widetilde{\mathcal{Y}}^{\xi}=\left(\widetilde{\mathcal{Y}}^{l, \xi}\right)_{0 \leq l \leq N}$ the corresponding sequence of solutions, with $\widetilde{\mathcal{Y}}^{l, \xi}=\left(\widetilde{Y}^{l, \xi}, \widetilde{Z}^{l, \xi}, \widetilde{A}^{l, \xi}\right)$ and $\widetilde{A}^{l, \xi}$ continuous, for every $l=0, \ldots, N$.

Proposition 2.6 (Prop. 14.2.14 in [8]; see also [13,16,18, 19,22]).

We assume $(\mathrm{H} \ell)$ and $(\mathrm{Hh})$. Then, for every $l=N, \ldots, 0$ :

(i) One has the following bound estimate on $\mathcal{Y}^{l, \xi}$,

$$
\left\|Y^{l, \xi}\right\|_{\mathcal{S}^{2}}^{2}+\left\|Z^{l, \xi}\right\|_{\mathcal{H}_{q}^{2}}^{2}+\left\|A^{l, \xi}\right\|_{\mathcal{S}^{2}}^{2} \leq C\left(1+|x|^{2 q}\right) .
$$

Moreover, an analogous bound estimate is satisfied by $\tilde{\mathcal{Y}}^{l, \xi}$;

(ii) Let $\xi_{n}=\left(t_{n}, x_{n}, k\right) \rightarrow \xi=(t, x, k)$ in $\mathcal{E}$ as $n \rightarrow \infty$.

- In case $t \notin \mathfrak{T}$, or in case $t=T_{I}$ and the $\xi_{n}$ are in $\mathcal{E}_{I+1}$, then there exists an extraction $\left(\xi_{n^{\prime}}\right)_{n}$ such that $\mathcal{Y}^{l, \xi_{n^{\prime}}}$ converges in $\mathcal{S}^{2} \times \mathcal{H}_{q}^{2} \times \mathcal{S}^{2}$ to $\mathcal{Y}^{l, \xi}$ as $n \rightarrow \infty$;

- In case $t=T_{I}, x \notin \partial \mathcal{O}$ and the $\xi_{n}$ are in $\mathcal{E}_{I}^{*}$, then there exists an extraction $\left(\xi_{n^{\prime}}\right)_{n}$ for which $\mathcal{Y}^{l, \xi_{n^{\prime}}}$ converges in $\mathcal{S}^{2} \times \mathcal{H}_{q}^{2} \times \mathcal{S}^{2}$ to $\widetilde{\mathcal{Y}}^{l, \xi}$ as $n \rightarrow \infty$.

Remark 2.7. In [8], an almost surely càdlàg property of $\vartheta$ slightly stronger than that established in Proposition 2.4, is postulated (see Assumption 14.2.12 in [8]). However, as easily seen by inspection of the proof in [8], the "sequential càdlàg property up to extraction of a subsequence" of Proposition 2.4 is enough for Proposition 2.6 to hold.

\subsection{Connection with finance}

In the case of risk-neutral pricing problems in finance, the driver coefficient function $f$ of $(\mathcal{E})$ is typically given as

$$
f=f(t, x, y)=c(t, x)-\mu(t, x) y,
$$

for dividend and interest-rate related functions $c$ and $\mu$. So $f$ is affine in $y$ and does not depend on $z$. Moreover, in the financial interpretation:

- $g\left(X_{T}\right)$ corresponds to a terminal payoff that is paid by the issuer to the holder at time $T$ if the contract was not exercised before $T$;

- $\ell\left(t, X_{t}\right)$, respectively $h\left(t, X_{t}\right)$, corresponds to a lower, respectively upper payoff that is paid by the issuer to the holder of the claim in the event of early termination of the contract at the initiative of the holder, respectively issuer;

- The sequence of stopping time $\vartheta$ is interpreted as a sequence of times of switching of a call protection. More precisely, the issuer of the claim is allowed to call it back, enforcing early exercise, on the "odd" (random) time intervals $\left[\vartheta_{2 l-1}, \vartheta_{2 l}\right)$. At other times call is not possible.

The contingent claims under consideration are thus general game contingent claims [20], covering convertible bonds, American options (and also European options) as special cases.

Now, in view of a rather standard verification principle and of the arbitrage theory for game options (see, e.g., [8]), if $\mathcal{Y}=(Y, Z, A)$ is a solution to $(\mathcal{E})$, then $\Pi=Y$ is an arbitrage price process for the game option, the arbitrage price relative to the pricing measure $\mathbb{P}$. Given a suitable set of hedging instruments, $\Pi$ is also a bilateral super-hedging price, in the sense that there exists a self-financing super-hedging strategy for the issuer of the claim starting from any issuer initial wealth greater than $\Pi$, and a self-financing super-hedging strategy for the holder of the claim starting from any holder initial wealth greater than $(-\Pi)$. Finally $\Pi$ is also the infimum of the initial wealths of all the issuer's self-financing super-hedging strategies. 
Note that modeling the pricing problem under the historical, as opposed to the risk-neutral, probability, would lead to a "z-dependent" driver coefficient function $f$. Also, the standard risk-neutral pricing approach tacitly assumes a perfect, frictionless financial market. Accounting for market imperfections would lead to a nonlinear coefficient $f$ (see, e.g., El Karoui et al. [14]).

A rather typical specification of the terminal cost functions is given by, for constants $\bar{P} \leq \bar{N} \leq \bar{C}$,

$$
\ell(t, x)=\bar{P} \vee S, h(t, x)=\bar{C} \vee S, g(x)=\bar{N} \vee S,
$$

where $S=x_{1}$ denotes the first component of $x$. Note that this specification satisfies assumptions (H $\left.\ell\right)-(\mathrm{Hh})$, as well as all the standing assumptions of this paper. In particular, one then has (cf. (1.1), (1.2) and (H $\ell)$ ),

$$
\lambda(t, x)=x_{1}=S, \mathcal{G} \lambda=b_{1},(\partial \lambda) \sigma=\sigma_{1},
$$

so that condition (2.1) in (Hh) reduces to $b_{1}$ and $\sigma_{1}$ being of class $(P)$, which holds by the Lipschitz property of $b$ and $\sigma$.

As for $\vartheta$, the following specifications are commonly found in the case of convertible bonds on an underlying stock $X=S$ (see $[8,10]$ for more details).

Example 2.8. Let the domain $\mathcal{O}$ be defined as $\left\{x \in \mathbb{R}^{q} \mid x_{1}<\bar{S}\right\}$, for some constant trigger level $\bar{S}$. Then, given a constant $l \leq N$, one may consider the following two specifications, where the first one can be seen as the special case where $d=l$ in the second one:

(i) $\mathcal{K}=\{0, \ldots, l\}, K=\{0, \ldots, l-1\}$, and, for every $I$,

$$
\kappa_{I}^{k}(x)= \begin{cases}(k+1) \wedge l, & x \notin \mathcal{O} \\ 0, & x \in \mathcal{O} .\end{cases}
$$

Starting from $H_{0}=0, H_{t}$ then represents the number of consecutive monitoring dates $T_{I}$ with $S_{T_{I}} \geq \bar{S}$ from time $t$ backwards, capped at $l$. Call is possible whenever $H_{t} \geq l$, which means that $S$ has been $\geq \bar{S}$ at the last $l$ monitoring times; otherwise call protection is in force.

(ii) $\mathcal{K}=\{0,1\}^{d}$ for some given integer $d \in\{l, \ldots, N\}, K=\left\{k \in \mathcal{K} ; \sum_{1 \leq p \leq d} k_{p}<l\right\}$, and, for every $I$,

$$
\kappa_{I}(x, k)=\left(k_{1}, \ldots, k_{d-1}, \mathbf{1}_{S \geq \bar{S}}\right)=\left\{\begin{array}{l}
\left(k_{1}, \ldots, k_{d-1}, 1\right), x \notin \mathcal{O} \\
\left(k_{1}, \ldots, k_{d-1}, 0\right), x \in \mathcal{O}
\end{array}\right.
$$

Starting from $H_{0}=(0, \ldots, 0) \in \mathbb{R}^{d}, H_{t}$ represents the vector of the indicator functions of the events $S_{T_{I}} \geq \bar{S}$ at the last $d$ monitoring dates preceding time $t$. Call is possible whenever $\sum_{1 \leq p \leq d} H_{t}^{p} \geq l$, which means that $S$ has been $\geq \bar{S}$ on at least $l$ of the last $d$ monitoring times; otherwise call protection is in force.

\section{VARIATIONAL INEQUALITIES APPROACH}

In view of introducing the value function $u$ related to our Markovian BSDE $(\mathcal{S})$, it is convenient to state the following definition.

\section{Definition 3.1.}

(i) A Cauchy cascade $\mathcal{U}$ on $\mathcal{E}$ is a sequence $\mathcal{U}=\left(u_{I}\right)_{1 \leq I \leq N}$ of functions $u_{I}$ of class $(P)$ on the $\mathcal{E}_{I}$, satisfying the following jump condition, at every $x \notin \partial \mathcal{O}$ :

$$
u_{I}^{k}\left(T_{I}, x\right)= \begin{cases}\min \left(u_{I+1}\left(T_{I}, x, \kappa_{I}^{k}(x)\right), h\left(T_{I}, x\right)\right) & \text { if } k \notin K \text { and } \kappa_{I}^{k}(x) \in K, \\ u_{I+1}\left(T_{I}, x, \kappa_{I}^{k}(x)\right) & \text { else, }\end{cases}
$$

where, in case $I=N, u_{I+1}$ is to be understood as $g$.

A continuous Cauchy cascade is a Cauchy cascade with continuous ingredients $u_{I}$ on the $\mathcal{E}_{I}$, except maybe for discontinuities of the $u_{I}^{k}$ at the points $\left(T_{I}, x\right)$ with $x \in \partial \mathcal{O}$. 
(ii) The function defined by a Cauchy cascade is the function on $\mathcal{E}$ given as the concatenation on the $\mathcal{E}_{I}^{*}$ of the $u_{I}$, and by the terminal condition $g$ at $T$.

Now,

Proposition 3.2 (Thm. 14.2.18 in [8]).

Assuming $(\mathrm{H} \ell)$ and $(\mathrm{Hh})$, the state-process $Y$ of $\mathcal{Y}$ satisfies, $\mathbb{P}$-a.s.,

$$
Y_{t}=u\left(t, \mathcal{X}_{t}\right), t \in[0, T]
$$

for the deterministic function pricing function $u(t, x, k)=Y_{t}^{t, x, k}$. Moreover, $u$ is defined by a continuous Cauchy cascade $\mathcal{U}=\left(u_{I}\right)_{1 \leq I \leq N}$ on $\mathcal{E}$.

Remark 3.3. The analog of Remark 2.7 also applies here.

The next step consists in deriving an analytic characterization of the value function $u$, or, more precisely, of $\mathcal{U}=\left(u_{I}\right)_{1 \leq I \leq N}$, as the solution of a related analytic problem.

A technical difficulty comes from the potential discontinuity in $x$ of the functions $u_{I}^{k}$ on $\mathfrak{T} \times \partial \mathcal{O}$, unless of course one is in the trivial case where $\kappa_{I,+}^{k}=\kappa_{I,-}^{k}$. Our next goal is to characterize the Cauchy cascade $\mathcal{U}$ defining $u$ in terms of a suitable notion of discontinuous viscosity solutions (see [7]) to the following Cauchy cascade of variational inequalities:

For $I$ decreasing from $N$ to 1 ,

- At $t=T_{I}$, for every $k \in \mathcal{K}$ and $x \notin \partial \mathcal{O}$,

$$
u_{I}^{k}\left(T_{I}, x\right)= \begin{cases}\min \left(u_{I+1}\left(T_{I}, x, \kappa_{I}^{k}(x)\right), h\left(T_{I}, x\right)\right), & k \notin K \text { and } \kappa_{I}^{k}(x) \in K \\ u_{I+1}\left(T_{I}, x, \kappa_{I}^{k}(x)\right), & \text { else, }\end{cases}
$$

with $u_{I+1}$ in the sense of $g$ in case $I=N$;

- On the time interval $\left[T_{I-1}, T_{I}\right)$, for every $k \in \mathcal{K}$,

$$
\left\{\begin{array}{l}
\min \left(-\mathcal{G} u_{I}^{k}-f^{u_{I}^{k}}, u_{I}^{k}-\ell\right)=0, k \in K \\
\max \left(\min \left(-\mathcal{G} u_{I}^{k}-f^{u_{I}^{k}}, u_{I}^{k}-\ell\right), u_{I}^{k}-h\right)=0, k \notin K
\end{array}\right.
$$

where we denote, for any function $v=v(t, x)$,

$$
f^{v}=f^{v}(t, x)=f(t, x, v(t, x)) .
$$

By standard arguments, see e.g. $[2,8,13,16,18,19]$, Proposition 3.2 implies that every $u_{I}$ is a viscosity solution in the usual sense [7] of (3.4) on $\mathcal{E}_{I}^{*}$. Now, in view of characterizing $u_{I}$ as the unique solution in some viscosity sense to (3.3)-(3.4) on $\mathcal{E}_{I}$, one needs to investigate the behavior of $u_{I}$ at the parabolic boundary $T_{I} \times \mathbb{R}^{q} \times \mathcal{K}$ of $\mathcal{E}_{I}$, and to make precise the corresponding notion of boundary condition for $u_{I}$ in (3.3). Toward this end, let us introduce the notation $u_{N+1}^{ \pm} \equiv g$, and for every $I<N, k \in \mathcal{K}$, and $x \in \mathbb{R}^{q}$,

$$
\begin{aligned}
& u_{I+1}^{+, k}\left(T_{I}, x\right)= \begin{cases}\min \left(u_{I+1}\left(T_{I}, x, \kappa_{I,+}^{k}\right), h\left(T_{I}, x\right)\right), k \notin K \text { and } \kappa_{I,+}^{k} \in K \\
u_{I+1}\left(T_{I}, x, \kappa_{I,+}^{k}\right), & \text { else, }\end{cases} \\
& u_{I+1}^{-, k}\left(T_{I}, x\right)= \begin{cases}\min \left(u_{I+1}\left(T_{I}, x, \kappa_{I,-}^{k}\right), h\left(T_{I}, x\right)\right), & k \notin K \text { and } \kappa_{I,-}^{k} \in K \\
u_{I+1}\left(T_{I}, x, \kappa_{I,-}^{k}\right), & \text { else. }\end{cases}
\end{aligned}
$$


Note that the functions $u_{I+1}^{ \pm, k}$ are continuous in $x$ on the $\mathcal{E}_{I}^{*}$, and that (3.3) can equivalently be written as,

$$
u_{I}^{k}\left(T_{I}, x\right)=\left\{\begin{array}{l}
u_{I+1}^{+, k}\left(T_{I}, x\right), x \notin \overline{\mathcal{O}} \\
u_{I+1}^{-, k}\left(T_{I}, x\right), x \in \mathcal{O} .
\end{array}\right.
$$

Let also for $I \leq N$,

$$
\begin{aligned}
& \check{u}_{I+1}^{k}\left(T_{I}, x\right)= \begin{cases}u_{I+1}^{+, k}\left(T_{I}, x\right), & x \notin \overline{\mathcal{O}} \\
u_{I+1}^{+, k}\left(T_{I}, x\right) \vee u_{I+1}^{-, k}\left(T_{I}, x\right), & x \in \partial \mathcal{O} \\
u_{I+1}^{-, k}\left(T_{I}, x\right), & x \in \mathcal{O},\end{cases} \\
& \widehat{u}_{I+1}^{k}\left(T_{I}, x\right)= \begin{cases}u_{I+1}^{+, k}\left(T_{I}, x\right), & x \notin \overline{\mathcal{O}} \\
u_{I+1}^{+, k}\left(T_{I}, x\right) \wedge u_{I+1}^{-, k}\left(T_{I}, x\right), & x \in \partial \mathcal{O} \\
u_{I+1}^{-, k}\left(T_{I}, x\right), & x \in \mathcal{O} .\end{cases}
\end{aligned}
$$

In the following definitions of solutions $u_{I}$ to (3.3)-(3.4), one assumes that the function $u_{I+1}$, which sits implicitly via $u_{I+1}^{ \pm, k}$ in (3.6), is known and given, and continuous in the $x$ variable at $t=T_{I}$. We refer the reader to [7] for the classical notions of viscosity solutions which are embedded in the following definitions.

Definition 3.4. A locally bounded upper semi-continuous function, respectively locally bounded lower semicontinuous function, $\omega=\omega^{k}(t, x)$, of class $(P)$ on $\mathcal{E}_{I}$, is called a subsolution, respectively supersolution, of (3.3)(3.4) on $\mathcal{E}_{I}$, if and only if:

(i) $\omega$ is a viscosity subsolution, respectively supersolution, of (3.4) on $\mathcal{E}_{I}^{*}$;

(ii) $\omega \leq \check{u}_{I+1}$, respectively $\omega \geq \widehat{u}_{I+1}$, at $T_{I}$. In case $\omega \leq \widehat{u}_{I+1}$, respectively $\omega \geq \check{u}_{I+1}$, at $T_{I}$, $\omega$ is said to be a strong subsolution, respectively strong supersolution, of (3.3)-(3.4) on $\mathcal{E}_{I}$.

We also introduce the following additional assumption on the driver coefficient $f$ :

$(\mathbf{H} \eta)$ There exists, for every $R>0$, a nonnegative function $\eta_{R}$ continuous and null at 0 such that

$$
\left|f(t, x, y)-f\left(t, x^{\prime}, y\right)\right| \leq \eta_{R}\left(\left|x-x^{\prime}\right|\right)
$$

for any $t \in[0, T]$ and $x, x^{\prime} \in \mathbb{R}^{q}, y \in \mathbb{R}$ with $|x|,\left|x^{\prime}\right| \leq R$.

In virtue of a rather standard comparison principle that can be found for instance as Theorem 13.2.2 in [8] (see also $[2,8,13,16,18,19])$, it holds that:

(CP) Assuming $(\mathbf{H} \eta)$, we have $\mu \leq \nu$ on $\mathcal{E}_{I}$, for every strong subsolution $\mu$ and supersolution $\nu$ (resp. subsolution $\mu$ and strong supersolution $\nu$ ) of (3.3)-(3.4) on $\mathcal{E}_{I}$.

\subsection{Non-decreasing call protection}

For $\varepsilon \geq 0$, let $\mathcal{O}_{\varepsilon}=\left\{x \in \mathbb{R}^{q} \mid \mathrm{d}(x)<\varepsilon\right\}$, let $u_{\varepsilon}$ stand for the pricing function of the pricing problem corresponding to the dilated domain $\mathcal{O}_{\varepsilon}$ and other data unchanged, and let $\mathcal{U}_{\varepsilon}=\left(u_{\varepsilon, I}\right)_{1 \leq I \leq N}$ denote the associated Cauchy cascade of functions. We attach the index $\varepsilon$ to all the formerly introduced quantities, defined in reference to the dilated domain $\mathcal{O}_{\varepsilon}$. We will need to postulate below that the call protection satisfies the following monotonicity assumption with respect to dilation of the domain $\mathcal{O}$.

$(\mathbf{H m})$ One has on $\mathcal{E}$ :

(i) $u \leq u_{\varepsilon} \leq u_{\varepsilon^{\prime}}$ for every $\varepsilon^{\prime} \geq \varepsilon \geq 0$

(ii) $u_{\varepsilon}\left(T_{I}, x, \kappa_{I,+}^{k}\right) \leq u_{\varepsilon}\left(T_{I}, x, k\right) \leq u_{\varepsilon}\left(T_{I}, x, \kappa_{I,-}^{k}\right)$ for every $\varepsilon \geq 0$. 
Remark 3.5. By doubly reflected BSDEs comparison theorem, Assumption (Hm)(i), respectively (ii), holds if for every $l>0$ :

$$
\left[\vartheta_{2 l-1}^{\varepsilon^{\prime}}, \vartheta_{2 l}^{\varepsilon^{\prime}}\right) \subseteq\left[\vartheta_{2 l-1}^{\varepsilon}, \vartheta_{2 l}^{\varepsilon}\right) \subseteq\left[\vartheta_{2 l-1}, \vartheta_{2 l}\right) \text {, so that } U \leq U^{\varepsilon} \leq U^{\varepsilon^{\prime}},
$$

respectively (denoting here by a further superscript $(t, x, k)$ the initial condition of the marker process $H^{\varepsilon}$ corresponding to the dilated domain $\mathcal{O}_{\varepsilon}$ )

$$
\left[\vartheta_{2 l-1}^{\varepsilon ; t, x, \kappa_{I,-}^{k}}, \vartheta_{2 l}^{\varepsilon ; t, x, \kappa_{I,-}^{k}}\right) \subseteq\left[\vartheta_{2 l-1}^{\varepsilon ; t, x, k}, \vartheta_{2 l}^{\varepsilon ; t, x, k}\right) \subseteq\left[\vartheta_{2 l-1}^{\varepsilon ; t, x, \kappa_{I,+}^{k}}, \vartheta_{2 l}^{\varepsilon ; t, x, \kappa_{I,+}^{k}}\right),
$$

so that

$$
U^{\varepsilon ; t, x, \kappa_{I,+}^{k}} \leq U^{\varepsilon ; t, x, k} \leq U^{\varepsilon ; t, x, \kappa_{I,-}^{k}} .
$$

Example 3.6. By application of the above sufficiency conditions, Assumption (Hm) holds for instance in the situations of Example 2.8. Let us thus consider the situation of Example 2.8(ii), which includes that of Example 2.8(i) as a special case. In view of the interpretation of $H_{t}$ as vector of the indicator functions of the events $S_{T_{I}} \geq \bar{S}$ at the last $d$ monitoring dates preceding time $t$, it follows for $\varepsilon^{\prime} \geq \varepsilon \geq 0$ that $\left|H^{\varepsilon^{\prime}}\right| \leq\left|H^{\varepsilon}\right| \leq|H|$, hence (3.9) follows. Moreover, we have in view of the definition (2.9) of the jump function in this example that for every $\varepsilon \geq 0$,

$$
\left|H^{\varepsilon ; t, x, \kappa_{I,-}^{k}}\right| \leq\left|H^{\varepsilon ; t, x, k}\right| \leq\left|H^{\varepsilon ; t, x, \kappa_{I,+}^{k}}\right|
$$

hence (3.10) follows.

Note that under $(\mathrm{Hm})(\mathrm{ii})$, the extensions to $\varepsilon \geq 0$ of definitions (3.7) are equivalent to

$$
\check{u}_{\varepsilon, I+1}^{k}\left(T_{I}, x\right)=\left\{\begin{array}{l}
u_{\varepsilon, I+1}^{+, k}\left(T_{I}, x\right), x \notin \overline{\mathcal{O}}_{\varepsilon} \\
u_{\varepsilon, I+1}^{-, k}\left(T_{I}, x\right), x \in \overline{\mathcal{O}}_{\varepsilon}
\end{array}, \widehat{u}_{\varepsilon, I+1}^{k}\left(T_{I}, x\right)=\left\{\begin{array}{l}
u_{\varepsilon, I+1}^{+, k}\left(T_{I}, x\right), x \notin \mathcal{O}_{\varepsilon} \\
u_{\varepsilon, I+1}^{-, k}\left(T_{I}, x\right), x \in \mathcal{O}_{\varepsilon}
\end{array}\right.\right.
$$

The following result establishes the convergence of the value function $u_{\varepsilon}$ for the dilated domain $\mathcal{O}_{\varepsilon}$ to the value function $u$, at the "regular" points $\xi=(t, x, k)$ with $(t, x) \notin \mathcal{T} \times \partial \mathcal{O}$.

Proposition 3.7. Assuming $(\mathrm{H} \ell),(\mathrm{Hh})$ and $(\mathrm{Hm})$, one has for every $(t, x, k) \in \mathcal{E}_{I}^{*}$

$$
\lim \searrow \epsilon \backslash 0+u_{\varepsilon, I}^{k}(t, x)=u_{I}^{k}(t, x) .
$$

Moreover the pointwise convergence (3.12) is uniform on every compact set of $\mathcal{E}_{I}^{*}$.

Let $\bar{u}_{\varepsilon, I}$, respectively $\underline{u}_{\varepsilon, I}$, denote the function on $\mathcal{E}_{I}$ defined as $u_{\varepsilon, I}$ on $\mathcal{E}_{I}^{*}$ and prolongated at $T_{I}$ by $\check{u}_{\varepsilon, I+1}$, respectively $\widehat{u}_{\varepsilon, I+1}$. Let also $\underline{u}_{I}=\underline{u}_{0, I}, \bar{u}_{I}=\bar{u}_{0, I}$.

The next result shows that every $u_{I}$ on $\mathcal{E}_{I}^{*}$, extended as $\bar{u}_{I}$ on $\mathcal{E}_{I}$, is the unique solution in some sense (maximal subsolution) to (3.3)-(3.4), in which $u_{I+1}$ determines the terminal condition at time $T_{I}$ (with $u_{N+1} \equiv g$ ). This result thus provides an analytical characterization of the value function $u$, in terms of the related Cauchy cascade of variational inequalities,

Theorem 3.8. Assuming $(\mathrm{H} \ell),(\mathrm{Hh}),(\mathrm{Hm})$ and $(\mathrm{H} \eta)$, one has for I decreasing from $N$ to 1 , pointwise on $\mathcal{E}_{I}$,

$$
\bar{u}_{I}=\lim \searrow_{\epsilon} 0+\underline{u}_{\varepsilon, I},
$$

and $\bar{u}_{I}$ is the largest subsolution of (3.3)-(3.4) on $\mathcal{E}_{I}$. 


\subsection{Deterministic approximation scheme}

We now discuss the numerical solution of the Cauchy cascade of variational inequalities (3.3)-(3.4), which, given the representation $(3.2)$, can be seen as a first way of solving $(\mathcal{S})$ numerically (at least, as far as determination of the value component $Y$ is concerned).

We work under assumption $(\mathrm{Hm})$. As we did above, we shall proceed iteratively in $I$ decreasing from $N$ to 1 . More specifically, we assume that the function $u_{I+1}$ which sits implicitly in $u_{I+1}^{ \pm, k}$ in (3.6), and more generally, every function $u_{\varepsilon, I+1}$ sitting implicitly in $u_{\varepsilon, I+1}^{ \pm, k}$ in (3.15) below for any $\varepsilon \geq 0$, is known and given. We then consider the problem of computing $u_{I}$, or, equivalently, its "upper semicontinuous envelope" $\bar{u}_{I}$, which was characterized analytically in Theorem 3.8 as the largest subsolution of (3.3)-(3.4) on $\mathcal{E}_{I}$.

Let $\left(U_{\varepsilon, I}^{\mathrm{h}}\right)^{\mathrm{h}>0}$, where $\varepsilon$ stands for the parameter of dilation of the domain $\mathcal{O}$ and $\mathrm{h}$ is a discretization parameter, denote a stable, monotone and consistent approximation scheme for $u_{\varepsilon, I}$, for every $\varepsilon>0$. On the notions of stable, monotone and consistent approximation scheme, we refer the reader to the seminal paper of Barles and Souganidis [2] and, as the closest reference to the present set-up, Subsection 13.2.3 of [8]. One may thus view $\left(U_{\varepsilon, I}^{\mathrm{h}}\right)^{\mathrm{h}>0}$ as the solution, suitably interpolated over $\mathcal{E}_{I}$, of a standard finite differences schemes for "the solution" ${ }^{6} u_{\varepsilon, I}$ to the following problem on $\mathcal{E}_{I}$ (cf. (3.3), (3.4) and (3.6), (3.11)):

- At $t=T_{I}$, for every $k \in \mathcal{K}$ and $x \notin \partial \mathcal{O}$,

$$
u_{\varepsilon, I}^{k}\left(T_{I}, x\right)=\left\{\begin{array}{l}
u_{\varepsilon, I+1}^{+, k}\left(T_{I}, x\right), x \notin \overline{\mathcal{O}}_{\varepsilon} \\
u_{\varepsilon, I+1}^{-, k}\left(T_{I}, x\right), x \in \mathcal{O}_{\varepsilon}
\end{array}\right.
$$

- On the time interval $\left[T_{I-1}, T_{I}\right)$, for every $k \in \mathcal{K}$,

$$
\left\{\begin{array}{l}
\min \left(-\mathcal{G} u_{\varepsilon, I}^{k}-f^{u_{\varepsilon, I}^{k}}, u_{\varepsilon, I}^{k}-\ell\right)=0, k \in K \\
\max \left(\min \left(-\mathcal{G} u_{\varepsilon, I}^{k}-f^{u_{\varepsilon, I}^{k}}, u_{\varepsilon, I}^{k}-\ell\right), u_{\varepsilon, I}^{k}-h\right)=0, k \notin K .
\end{array}\right.
$$

One also refers the reader to [2] or Subsection 13.2.3 of [8], for the classical notions of lower and upper envelopes of the numerical scheme $\left(U_{\varepsilon, I}^{\mathrm{h}}\right)^{\mathrm{h}>0}$ as $\mathrm{h} \rightarrow 0^{+}$(for a fixed $\varepsilon>0$, here). Building on these notions, one then has the following "double convergence" result.

Proposition 3.9. We assume $(\mathrm{H} \ell),(\mathrm{Hh}),(\mathrm{Hm})$ and $(\mathrm{H} \eta)$.

(i) For every $\varepsilon>0$, one has on $\mathcal{E}_{I}$,

$$
\bar{u}_{I} \leq \underline{U}_{\varepsilon, I} \leq \bar{U}_{\varepsilon, I} \leq \underline{u}_{2 \varepsilon, I},
$$

where $\underline{U}_{\varepsilon, I}$ and $\bar{U}_{\varepsilon, I}$ denote the lower and upper envelopes of the numerical scheme $\left(U_{\varepsilon, I}^{\mathrm{h}}\right)^{\mathrm{h}>0}$.

(ii) As $\varepsilon \rightarrow 0^{+}$, the double scheme $\left(U_{\varepsilon, I}^{\mathrm{h}}\right)_{\varepsilon>0}^{\mathrm{h}>0}$ converges to $u_{I}$ locally uniformly on $\mathcal{E}_{I}^{*}$, in the sense that one has for every compact set $\mathcal{C}$ of $\mathcal{E}_{I}^{*}$, for every $\gamma>0$ :

$$
\max _{\mathcal{C}}\left|U_{\varepsilon, I}^{\mathrm{h}}-u_{I}\right| \leq \gamma
$$

for $\varepsilon<\varepsilon(\gamma)$ and $\mathrm{h}<\mathrm{h}(\varepsilon)$.

Note that this proposition only yields a partial convergence result, since one does not know the functions $\varepsilon(\gamma)$ and $\mathrm{h}(\varepsilon)$ in Proposition 3.9(ii). Moreover, one only gets the convergence on $\mathcal{E}_{I}^{*}$ under the working assumption that the true value for $u_{\varepsilon, I+1}$ is plugged at $T_{I}$ in the approximation schemes (3.14)-(3.15) for $u_{\varepsilon, I}$. In this regard this result remains a bit theoretical.

\footnotetext{
${ }^{6}$ In any reasonable meaning, e.g., $\bar{u}_{\varepsilon, I}$ largest viscosity subsolution.
} 
It is also theoretical in the sense that (3.14)-(3.15) involves $\operatorname{Card}(\mathcal{K})$ equations in the $u_{\varepsilon, I}^{k}$. From a deterministic computational point of view, the Cauchy cascade (3.14)-(3.15) (or (3.3)-(3.4)) can thus be considered as a " $q+d$ - dimensional" pricing problem, with $d=\log (\operatorname{Card}(\mathcal{K}))$. For "very large" sets $\mathcal{K}$, like for instance in Example 2.8(ii), the use of deterministic schemes is precluded by the curse of dimensionality, and simulation schemes such as the one of the next section are the only viable alternative. We refer the reader to [10] for a thorough comparison of the practical performances of the two schemes.

\section{RIBSDE TIME-DISCRETIZATION RESULTS}

In Sections 4.1 to 4.3, we propose an approximation scheme in time for a solution $\mathcal{Y}=(Y, Z, A)$, assumed to exist, to $(\mathcal{S}$ ) (for instance because assumption $(\mathrm{H} \ell$ ) holds, see Prop. 2.3), and we provide an upper bound for the convergence rate of this scheme.

For every time-grid $\mathfrak{t}:=\left\{0=t_{0}<t_{1}<\ldots<t_{n}=T\right\}$ of $[0, T]$ we denote

$$
|\mathfrak{t}|=\max _{i \leq n-1}\left(t_{i+1}-t_{i}\right),|\mathfrak{t}|^{\sharp}=\min _{i \leq n-1}\left(t_{i+1}-t_{i}\right) .
$$

In the sequel, we consider a particular time-grid $\mathfrak{t}:=\left\{0=t_{0}<t_{1}<\ldots<t_{n}=T\right\}$ of $[0, T]$, which will be used for time-discretizing the RIBSDE $(\mathcal{S})$.

\subsection{Approximation of the forward process}

When the diffusion $X$ in (1.1) cannot be simulated exactly, we use the Euler approximation scheme $\widehat{X}$ defined on the time-grid $\mathfrak{t}$, by $\widehat{X}_{0}=X_{0}$, and for $i \leq n-1$,

$$
\widehat{X}_{t_{i+1}}=\widehat{X}_{t_{i}}+b\left(t_{i}, \widehat{X}_{t_{i}}\right)\left(t_{i+1}-t_{i}\right)+\sigma\left(t_{i}, \widehat{X}_{t_{i}}\right)\left(W_{t_{i+1}}-W_{t_{i}}\right) \text {. }
$$

We assume $n|\mathfrak{t}| \leq \Lambda$. We also define the usual continuous-time extension of $\widehat{X}$ by setting, for every $i \leq n-1$ and $t \in\left[t_{i}, t_{i+1}\right)$,

$$
\widehat{X}_{t}=\widehat{X}_{t_{i}}+b\left(t_{i}, \widehat{X}_{t_{i}}\right)\left(t-t_{i}\right)+\sigma\left(t_{i}, \widehat{X}_{t_{i}}\right)\left(W_{t}-W_{t_{i}}\right)
$$

or in an equivalent differential notation, for $t \in[0, T]$,

$$
\mathrm{d} \widehat{X}_{t}=\mathrm{b}\left(\bar{t}, \widehat{X}_{\bar{t}}\right) \mathrm{d} t+\sigma\left(\bar{t}, \widehat{X}_{\bar{t}}\right) \mathrm{d} W_{t}
$$

with $\bar{t}:=\sup \{s \in \mathfrak{t} \mid s \leq t\}$. Under the Lipschitz continuity assumption $(\mathrm{H} x)$, one has, for every $p \geq 1$, see e.g. [21],

$$
\left\|\sup _{t \leq T}\left|X_{t}-\widehat{X}_{t}\right|\right\|_{\mathcal{L}^{p}}+\max _{i<n}\left\|\sup _{t \in\left[t_{i}, t_{i+1}\right]}\left|X_{t}-\widehat{X}_{t_{i}}\right|\right\|_{\mathcal{L}^{p}} \leq C_{\Lambda}^{p}|\mathfrak{t}|^{\frac{1}{2}}
$$

\subsection{Approximation of the Barriers}

The lower barrier is approximated by $\widehat{L}_{t}=\ell\left(t, \widehat{X}_{t}\right)$. As for the upper barrier, we first define the approximation $\widehat{H}$ of the marker process $H$ by

$$
\widehat{H}_{0}=H_{0} \text { and } \widehat{H}_{T_{I}}=\kappa_{I}\left(\widehat{X}_{T_{I}}, \widehat{H}_{T_{I}-}\right), \text { for } 1 \leq I \leq N .
$$

We then define the approximation $\widehat{\vartheta}$ of $\vartheta$ as the sequence of $\mathfrak{T}$-valued stopping times obtained by using $\widehat{\mathcal{X}}=(\widehat{X}, \widehat{H})$ instead of $\mathcal{X}$ in (1.5). This leads to the following approximation of the upper boundary:

$$
\widehat{U}_{t}=\sum_{l=0}^{[N / 2]} \mathbf{1}_{\left[\widehat{\vartheta}_{2 l}, \widehat{\vartheta}_{2 l+1}\right)} \infty+\sum_{l=1}^{[(N+1) / 2]} \mathbf{1}_{\left[\widehat{\vartheta}_{2 l-1}, \widehat{\vartheta}_{2 l}\right)} h\left(t, \widehat{X}_{t}\right) .
$$

The following control is key in the sequel. 
Proposition 4.1. For every $\epsilon>0$, there exists a constant $C_{\Lambda}^{\epsilon}$ such that for every $l \leq N+1$,

$$
\mathbb{E}\left[\left|\vartheta_{l}-\widehat{\vartheta}_{l}\right|\right] \leq C_{\Lambda}^{\epsilon}|\mathfrak{t}|^{\frac{1}{2}-\epsilon}
$$

\subsection{Approximation of the RIBSDE}

In the sequel, we shall use one of the following regularity assumptions:

(Hb) $h$ and $\ell$ are $\Lambda$-Lipschitz continuous with respect to $(t, x)$,

(Hb)' $h$ and $\ell$ are $\Lambda$-Lipschitz continuous with respect to $(t, x)$ and there exists a constant $\Lambda$ and some functions $\Lambda_{1}, \Lambda_{2}: \mathbb{R}^{q} \rightarrow \mathbb{R}^{q}$ and $\Lambda_{3}: \mathbb{R}^{q} \rightarrow \mathbb{R}^{+}$such that $\left|\Lambda_{1}(x)\right|+\left|\Lambda_{2}(x)\right|+\left|\Lambda_{3}(x)\right| \leq \Lambda\left(1+|x|^{\Lambda}\right)$, and for every $x, y \in \mathbb{R}^{q}$,

$$
\begin{aligned}
& \ell(t, x)-\ell(t, y) \leq \Lambda_{1}(x)(y-x)+\Lambda_{3}(x)|x-y|^{2} \\
& h(t, y)-h(t, x) \leq \Lambda_{2}(x)(y-x)+\Lambda_{3}(x)|x-y|^{2} .
\end{aligned}
$$

Note that assumption $(\mathrm{Hb})^{\prime}$ is related to the classical semi-convexity assumption of Definition 1 in [1]. For $\varrho$ defined as $\vartheta$ or $\widehat{\vartheta}$, let the projection operator $\mathcal{P}_{\varrho}$ be defined by

$$
\mathcal{P}_{\varrho}(t, x, y)=y+[\ell(t, x)-y]^{+}-[y-h(t, x)]^{+} \sum_{l=1}^{[(N+1) / 2]} \mathbf{1}_{\left\{\varrho_{2 l-1} \leq t \leq \varrho_{2 l}\right\}} .
$$

To tackle the reflection issue, we introduce a discrete set of reflection times defined by

$$
\mathfrak{r}=\left\{0=r_{0}<r_{1}<\ldots<r_{\nu}=T\right\},
$$

such that $\mathfrak{T} \subseteq \mathfrak{r} \subseteq \mathfrak{t}$ and $|\mathfrak{r}| \leq C_{\Lambda}|\mathfrak{r}|^{\sharp}$, where $|\mathfrak{r}|^{\sharp}=\min _{j \leq \nu-1}\left(r_{j+1}-r_{j}\right)$. Here the point is that, in the approximation scheme for $\mathcal{Y}$, the reflection will operate only on $\mathfrak{r}$ (this feature is used in the Proof of Thm. 4.3, see end of Sect. 5.2.4). The components $Y$ and $Z$ of a solution $\mathcal{Y}=(Y, Z, A)$ to the RIBSDE $(\mathcal{S})$ are thus approximated by a triplet of processes $(\widehat{Y}, \widetilde{Y}, \bar{Z})$ on $\mathfrak{t}$, which are defined by the terminal condition

$$
\widehat{Y}_{T}=\widetilde{Y}_{T}=g\left(\widehat{X}_{T}\right),
$$

and then satisfy the following relations, for $i$ decreasing from $n-1$ to 0 :

$$
\left\{\begin{array}{l}
\bar{Z}_{t_{i}}=\frac{1}{t_{i+1}-t_{i}} \mathbb{E}_{t_{i}}\left[\widehat{Y}_{t_{i+1}}\left(W_{t_{i+1}}-W_{t_{i}}\right)^{\prime}\right] \\
\widetilde{Y}_{t_{i}}=\mathbb{E}_{t_{i}}\left[\widehat{Y}_{t_{i+1}}\right]+\left(t_{i+1}-t_{i}\right) f\left(t_{i}, \widehat{X}_{t_{i}}, \widetilde{Y}_{t_{i}}\right) \\
\widehat{Y}_{t_{i}}=\widetilde{Y}_{t_{i}} \mathbf{1}_{\left\{t_{i} \notin \mathfrak{r}\right\}}+\mathcal{P}_{\widehat{\vartheta}}\left(t_{i}, \widehat{X}_{t_{i}}, \widetilde{Y}_{t_{i}}\right) \mathbf{1}_{\left\{t_{i} \in \mathfrak{r}\right\}} .
\end{array}\right.
$$

By convention, we also set $\bar{Z}_{T}=0$. Using an induction argument and the Lipschitz-continuity assumption on $f, g, l, h$, one easily checks that the above processes are square integrable. It follows that the conditional expectations are well defined at each step of the algorithm.

We also need for the proofs a piecewise time-continuous extension of the scheme. Using the martingale representation theorem, we define $\widehat{Z}$ on $\left[t_{i}, t_{i+1}\right)$ by

$$
\widehat{Y}_{t_{i+1}}=\mathbb{E}_{t_{i}}\left[\widehat{Y}_{t_{i+1}}\right]+\int_{t_{i}}^{t_{i+1}} \widehat{Z}_{s} \mathrm{~d} W_{s} .
$$

We then define $\tilde{Y}$ on $\left[t_{i}, t_{i+1}\right)$ by

$$
\widetilde{Y}_{t}=\widehat{Y}_{t_{i+1}}+\left(t_{i+1}-t\right) f\left(t_{i}, \widehat{X}_{t_{i}}, \widetilde{Y}_{t_{i}}\right)-\int_{t}^{t_{i+1}} \widehat{Z}_{s} \mathrm{~d} W_{s}
$$


and we let finally, for $t \in[0, T]$,

$$
\widehat{Y}_{t}=\widetilde{Y}_{t} \mathbf{1}_{\{t \notin \mathfrak{r}\}}+\mathcal{P}_{\widehat{\vartheta}}\left(t, \widehat{X}_{t}, \widetilde{Y}_{t}\right) \mathbf{1}_{\{t \in \mathfrak{r}\}} .
$$

Observe that one has, for $i \leq n-1$,

$$
\bar{Z}_{t_{i}}=\frac{1}{t_{i+1}-t_{i}} \mathbb{E}_{t_{i}}\left[\int_{t_{i}}^{t_{i+1}} \widehat{Z}_{s} \mathrm{~d} s\right]
$$

We also define $\bar{Z}_{t}=\bar{Z}_{\bar{t}}$, for $t \in[0, T]$.

\subsubsection{Convergence results}

When there is no call or no call protection, the convergence of the scheme is given by Theorem 6.2 in [6] and Theorem 4.1 in [3] (in which $f$ may actually further depend on $z$ ).

Theorem 4.2 (see $[3,6]$ ). We assume no call or no call protection. With $\alpha=\frac{1}{3}$ and $|\mathfrak{r}| \sim|\mathfrak{t}|^{\frac{2}{3}}$ under $(\mathrm{Hb})$, respectively $\alpha=\frac{1}{2}$ and $|\mathfrak{r}| \sim|\mathfrak{t}|^{\frac{1}{2}}$ under $(\mathrm{Hb})^{\prime}$, one has,

$$
\max _{i \leq n-1} \sup _{t \in\left[t_{i}, t_{i+1}\right)} \mathbb{E}\left[\left|Y_{t}-\widetilde{Y}_{t_{i}}\right|^{2}+\left|Y_{t}-\widehat{Y}_{t_{i}}\right|^{2}\right]+\mathbb{E}\left[\int_{0}^{T}\left|Z_{t}-\bar{Z}_{\bar{t}}^{2}\right| \mathrm{d} t\right] \leq C_{\Lambda}|\mathfrak{t}|^{\alpha} .
$$

Note that under stronger assumption on the boundaries and on the regularity of the coefficients $b, \sigma$, it is possible to obtain a better control of the convergence rate of the approximation, see Theorem 6.2 in [6] and Theorem 4.1 in [3].

Regarding call protection, our main result is the following.

Theorem 4.3. With $\alpha=\frac{1}{4}$ and $|\mathfrak{r}| \sim|\mathfrak{t}|^{\frac{1}{2}}$ under $(\mathrm{Hb})$, respectively $\alpha=\frac{1}{2}$ and $\mathfrak{r}=\mathfrak{t}$ under $(\mathrm{Hb})^{\prime}$, one has,

$$
\max _{i \leq n-1} \sup _{t \in\left[t_{i}, t_{i+1}\right)} \mathbb{E}\left[\left|Y_{t}-\widetilde{Y}_{t_{i}}\right|^{2}\right]+\max _{i \leq n-1} \sup _{t \in\left[t_{i}, t_{i+1}\right)} \mathbb{E}\left[\left|Y_{t-}-\widehat{Y}_{t_{i}}\right|^{2}\right] \leq C_{\Lambda}^{\epsilon}|\mathfrak{t}|^{\alpha-\epsilon},
$$

for every $\epsilon>0$.

We also have the following result concerning the approximation of the $Z$-process in the general case.

Theorem 4.4. Under $(\mathrm{Hb})$, setting $|\mathfrak{r}| \sim|\mathfrak{t}|^{\frac{1}{2}}$, we have

$$
\lim _{|\mathfrak{t}| \rightarrow 0} \mathbb{E}\left[\int_{0}^{T}\left|Z_{t}-\bar{Z}_{\bar{t}}^{2}\right| \mathrm{d} t\right]=0
$$

Remark 4.5. As shown in Theorem 4.2, in the "no call" or "no call protection" cases, convergence bounds are also available for $Z$, see Theorem 6.1 in [6] and Theorem 4.1 in [3]. The "call protection" case is currently an open problem in this regard, as more generally in regard to establishing convergence bounds on $Y$ and $Z$ in case $f$ depends on $z$. Maybe this could be dealt with by combining the ideas of the present work with the techniques of Gobet and Makhlouf [15]. We leave this for further research however.

\subsubsection{Discretely reflected BSDEs}

The Proof of Theorem 4.3 will be done in Section 5 in several steps, using a suitable concept of a discretely reflected BSDE. In finance, discretely reflected BSDEs represent game option which can be exercised only on the discrete set of times $\mathfrak{r}$. 
Given the reflection grid $\mathfrak{r}$ as of (4.6) and for $\varrho=\vartheta$ or $\widehat{\vartheta}$, the solution of the discretely reflected BSDE is a triplet $\left(\mathfrak{I}^{\varrho}, \widetilde{\mathfrak{I}}^{\varrho}, \mathfrak{Z}^{\varrho}\right)$ defined by the terminal condition

$$
\mathfrak{I}_{T}^{\varrho}=\widetilde{\mathfrak{I}}_{T}^{\varrho}=g\left(X_{T}\right),
$$

and then for $\imath$ decreasing from $\nu-1$ to 0 in (4.6) and $t \in\left[r_{\imath}, r_{\imath+1}\right)$,

$$
\left\{\begin{array}{l}
\widetilde{\mathfrak{I}}_{t}^{\varrho}=\mathfrak{I}_{r_{i+1}}^{\varrho}+\int_{t}^{r_{i+1}} f\left(u, X_{u}, \widetilde{\mathfrak{I}}_{u}^{\varrho}\right) \mathrm{d} u-\int_{t}^{r_{\imath+1}} \mathfrak{Z}_{u}^{\varrho} \mathrm{d} W_{u}, \\
\mathfrak{I}_{t}^{\varrho}=\widetilde{\mathfrak{I}}_{t}^{\varrho} \mathbf{1}_{\{t \notin \mathfrak{r}\}}+\mathcal{P}_{\varrho}\left(t, X_{t}, \widetilde{\mathfrak{I}}_{t}^{\varrho}\right) \mathbf{1}_{\{t \in \mathfrak{r}\}} .
\end{array}\right.
$$

Under $(\mathrm{Hb})$, the triplet $\left(\mathfrak{I}^{\varrho}, \widetilde{\mathfrak{I}}^{\varrho}, \mathfrak{Z}^{\varrho}\right)$ can be defined by backward induction. At each step, existence and uniqueness of a solution in $\mathcal{S}^{2} \times \mathcal{H}_{q}^{2}$ follow from [14]. Note that $\widetilde{\mathfrak{I}}^{\varrho}$ is a càdlàg process, whereas $\mathfrak{I}^{\varrho}$ is a càglàd process. Also observe that one has, for $r \in \mathfrak{r}$,

$$
Y_{r-}=\mathcal{P}_{\vartheta}\left(r, X_{r}, Y_{r}\right), \mathfrak{I}_{r}^{\varrho}=\mathcal{P}_{\varrho}\left(r, X_{r}, \widetilde{\mathfrak{I}}_{r}^{\varrho}\right) .
$$

We first present two properties of discretely reflected BSDEs which are useful to prove Theorem 4.3. We show that under suitable conditions the discretely reflected BSDE with $\varrho=\vartheta$ is a "good" approximation of the $\operatorname{RIBSDE}(\mathcal{E})$. In view of Definition 2.2(i), the component $Y$ of $\mathcal{Y}$ may be discontinuous at $\vartheta_{2 l}$ on $0<\vartheta_{2 l}<T$. The fact that $\mathfrak{T} \subseteq \mathfrak{r}$ will then be essential to obtain the following result.

Proposition 4.6. Let $\alpha=\frac{1}{2}$ or $\alpha=1$ under $(\mathrm{Hb})$ or $(\mathrm{Hb})$ ', respectively. Then,

$$
\sup _{t \in[0, T]} \mathbb{E}\left[\left|Y_{t}-\widetilde{\mathfrak{I}}_{t}^{\vartheta}\right|^{2}+\left|Y_{t-}-\Im_{t}^{\vartheta}\right|^{2}\right]+\mathbb{E}\left[\int_{0}^{T}\left|Z_{s}-\mathfrak{Z}_{s}^{\vartheta}\right|^{2} \mathrm{~d} s\right] \leq|\mathfrak{r}|^{\alpha}
$$

We also give a control of the difference between the solutions $\left(\mathfrak{I}^{\vartheta}, \widetilde{\mathfrak{I}}^{\vartheta}, \mathfrak{Z}^{\vartheta}\right)$ and $\left(\mathfrak{I}^{\widehat{\vartheta}}, \widetilde{\mathfrak{I}}^{\widehat{\vartheta}}, \mathfrak{\mathfrak { Z }}^{\widehat{\vartheta}}\right)$ of the two discretely reflected BSDEs with $\varrho=\vartheta$ and $\widehat{\vartheta}$.

Proposition 4.7. Let $\alpha=\frac{1}{2}$ or $\alpha=1$ under $(\mathrm{Hb})$ or $(\mathrm{Hb})$ ', respectively. Then,

$$
\sup _{t \in[0, T]} \mathbb{E}\left[\left|\mathfrak{I}_{t}^{\vartheta}-\mathfrak{I}_{t}^{\widehat{\vartheta}}\right|^{2}+\left|\widetilde{\mathfrak{I}}_{t}^{\vartheta}-\widetilde{\mathfrak{I}}_{t}^{\widehat{\vartheta}}\right|^{2}\right]+\mathbb{E}\left[\int_{0}^{T}\left|\mathfrak{Z}_{t}^{\vartheta}-\mathfrak{Z}_{t}^{\widehat{\vartheta}}\right|^{2} \mathrm{~d} t\right] \leq C_{\Lambda}^{\epsilon}|\mathfrak{r}|^{\alpha-1} \sum_{l=1}^{N}\left(\mathbb{E}\left[\left|\vartheta_{l}-\widehat{\vartheta}_{l}\right|\right]\right)^{1-\epsilon},
$$

for every $\epsilon>0$.

We conclude this section by giving a bound for the convergence rate of the scheme (4.7) to the discretely reflected BSDE (4.9), with $\varrho=\vartheta$.

Proposition 4.8. Let $\alpha=\frac{1}{2}$ or $\alpha=1$ under (Hb) or (Hb)'. Then,

$$
\sup _{t \in[0, T]} \mathbb{E}\left[\left|\widetilde{\mathfrak{I}}_{t}^{\vartheta}-\widetilde{Y}_{t}\right|^{2}\right]+\sup _{t \in[0, T]} \mathbb{E}\left[\left|\Im_{t}^{\vartheta}-\widehat{Y}_{t}\right|^{2}\right] \leq C_{\Lambda}|\mathfrak{t}|+C_{\Lambda}^{\epsilon}|\mathfrak{r}|^{\alpha-1} \sum_{l=1}^{N}\left(\mathbb{E}\left[\left|\vartheta_{l}-\widehat{\vartheta}_{l}\right|\right]\right)^{1-\epsilon}
$$

and

$$
\mathbb{E}\left[\int_{0}^{T}\left|\mathfrak{Z}_{t}^{\vartheta}-\widehat{Z}_{t}\right|^{2} \mathrm{~d} t\right] \leq C_{\Lambda} \nu|\mathfrak{t}|+C_{\Lambda}^{\epsilon}|\mathfrak{r}|^{\alpha-1} \sum_{l=1}^{N}\left(\mathbb{E}\left[\left|\vartheta_{l}-\widehat{\vartheta}_{l}\right|\right]\right)^{1-\epsilon}
$$

for $\epsilon>0$. 


\section{Proofs}

\subsection{Stability of call protection switching times}

Let us first define $\xi=(t, x, k)$ and $\xi^{\prime}=\left(t^{\prime}, x^{\prime}, k\right)$ for $\left(t, t^{\prime}\right) \in[0, T]^{2},\left(x, x^{\prime}\right) \in \mathbb{R}^{2 q}$ and $k \in \mathcal{K}$. In the following, we consider two Itô processes with different initial condition and coefficients. The first one, $X^{\xi}$, is the solution of the following SDE:

$$
X_{s}^{\xi}=x+\int_{t}^{s} b\left(s, X_{s}^{\xi}\right) \mathrm{d} s+\int_{t}^{s} \sigma\left(s, X_{s}^{\xi}\right) \mathrm{d} W_{s}, \quad \text { for } s \in[t, T] .
$$

The second one, $\check{X}^{\xi^{\prime}}$ can be written:

$$
\check{X}_{s}^{\xi^{\prime}}=x^{\prime}+\int_{t^{\prime}}^{s} \check{b}_{s} \mathrm{~d} s+\int_{t^{\prime}}^{s} \check{\sigma}_{s} \mathrm{~d} W_{s}, \quad \text { for } s \in\left[t^{\prime}, T\right] .
$$

We consider the following "monitoring grid" for $X^{\xi}$, respectively $\check{X}^{\xi}$ :

$$
\mathfrak{T}^{t}=\{s \in \mathfrak{T} \mid s>t\}, \quad \text { respectively } \mathfrak{T}^{t^{\prime}}=\left\{s \in \mathfrak{T} \mid s>t^{\prime}\right\},
$$

and we let $\mathcal{T}^{t}=\inf \mathfrak{T}^{t}$, respectively $\mathcal{T}^{t^{\prime}}=\inf \mathfrak{T}^{t^{\prime}}$.

Let us also introduce $\check{\mathcal{X}}^{\xi^{\prime}}=\left(\check{X}^{\xi^{\prime}}, \check{H}^{\xi^{\prime}}\right)$, where the marker process $\check{H}^{\xi^{\prime}}$ is defined by $\check{H}_{t^{\prime}}^{\xi^{\prime}}=k$, and for every $T_{I} \in \mathfrak{T}^{t^{\prime}}$

$$
\check{H}_{T_{I}}^{\xi^{\prime}}=\kappa_{I}\left(\check{X}_{T_{I}}^{\xi^{\prime}}, \check{H}_{T_{I}-}^{\xi^{\prime}}\right),
$$

and $\check{H}^{\xi^{\prime}}$ is constant between two dates of $\left\{t^{\prime}\right\} \cup \mathfrak{T}^{t^{\prime}}$. Observe that $\check{H}^{\xi^{\prime}}$ does not jump at $t^{\prime}$.

We also consider a non-decreasing sequence of stopping times $\breve{\vartheta}^{\prime}=\left(\breve{\vartheta}_{l}^{\xi^{\prime}}\right)_{0 \leq l \leq N+1}$, representing call protection switching times, defined by $\breve{\vartheta}_{0}^{\xi^{\prime}}=t^{\prime}$ and for every $l \geq 1$,

$$
\check{\vartheta}_{2 l+1}^{\xi^{\prime}}=\inf \left\{t>\check{\vartheta}_{2 l}^{\xi^{\prime}} ; H_{t}^{\prime} \notin K\right\} \wedge T, \check{\vartheta}_{2 l+2}^{\xi^{\prime}}=\inf \left\{t>\check{\vartheta}_{2 l+1}^{\xi^{\prime}} ; H_{t}^{\prime} \in K\right\} \wedge T .
$$

The $\breve{\vartheta}_{l}^{\xi^{\prime}}$ effectively reduce to $\left\{t^{\prime}\right\} \cup \mathfrak{T}^{t^{\prime}}$-valued stopping times, and one has $\check{\vartheta}_{N+1}^{\xi^{\prime}}=T$.

To the process $X^{\xi}$, we associate three different extended factor processes $\mathcal{X}^{\xi}, \mathcal{X}^{\xi, \varepsilon}$, for $\varepsilon>0$ and $\tilde{\mathcal{X}}^{\xi}$.

The first one, $\mathcal{X}^{\xi}=\left(X^{\xi}, H^{\xi}\right)$, is defined as above, replacing $\check{X}^{\xi^{\prime}}$ by $X^{\xi}$. Observe that $H^{\xi}$ does not jump at $t$ and that $H_{t}^{\xi}=\check{H}_{t^{\prime}}^{\xi^{\prime}}=k$. We also consider the sequence of call protection monitoring times $\vartheta$, defined as in (5.1) with $t$ and $H^{\xi}$ instead of $t^{\prime}$ and $\check{H}^{\xi^{\prime}}$.

The second factor process $\mathcal{X}^{\xi, \varepsilon}, \varepsilon>0$, is defined as $\mathcal{X}$ but using the dilated domain $\mathcal{O}_{\varepsilon}:=\left\{x \in \mathbb{R}^{q} \mid \mathrm{d}(x)<\varepsilon\right\}$ instead of $\mathcal{O}$ in the construction of $H^{\xi, \varepsilon}$, recalling Section 3.1. We also consider the sequence of call protection monitoring times $\vartheta^{\xi, \varepsilon}$, defined as in (5.1) with $t$ and $H^{\xi, \varepsilon}$ instead of $t^{\prime}$ and $\check{H}^{\xi^{\prime}}$.

The third factor process, $\widetilde{\mathcal{X}}^{\xi}=\left(X^{\xi}, \widetilde{H}^{\xi}\right)$, defined using the domain $\mathcal{O}$, is given by $\widetilde{H}_{t-}^{\xi}=k$, and for every $T_{I} \in \mathfrak{T}^{t^{\prime}}$

$$
\widetilde{H}_{T_{I}}^{\xi}=\kappa_{I}\left(T_{I}, \widetilde{H}_{T_{I}-}^{\xi}\right)
$$

and $\widetilde{H}$ constant between two dates of $\{t\} \cup \mathfrak{T}^{t}$. Observe that, contrary to $H^{\xi}, \widetilde{H}^{\xi}$ may jump at $t$. We also consider the corresponding call protection switching times $\widetilde{\vartheta}^{\xi}$ defined as in (5.1) with $t$ and $\widetilde{H}^{\xi}$ instead of $t^{\prime}$ and $\check{H}^{\xi^{\prime}}$.

We are interested in two different cases regarding the initial set of data $(t, x)$ and $\left(t^{\prime}, x^{\prime}\right)$.

Case 1. $\mathcal{T}^{t}=\mathcal{T}^{t^{\prime}}$.

Case 2. $\mathcal{T}^{t^{\prime}}=t$ and $x \notin \partial \mathcal{O}$.

The proof of the following Lemma is deferred to 5.3.3. 
Lemma 5.1. (i) One has, for $T_{I} \in \mathfrak{T}^{t}$,

$$
\mathbb{P}\left(\left\{\left|\mathrm{d}\left(X_{T_{I}}^{\xi}\right)\right| \leq \delta\right\}\right) \leq C_{\Lambda}^{\epsilon} \delta^{1-\epsilon}, \forall \epsilon>0 .
$$

(ii) For $p, \epsilon>0$, and $l=0, \ldots, N+1$, one has,

$$
\mathbb{E}\left[\mid \varrho_{l}-\check{\vartheta}_{l}^{\xi^{\prime}}\right] \leq\left|t-t^{\prime}\right|+C_{\Lambda}^{\epsilon} \delta^{1-\epsilon}+C_{\Lambda}^{p} \frac{\mathbb{E}\left[\sup _{u \in\left[\mathcal{T}^{t}, T\right]}\left|\check{X}_{u}^{\xi^{\prime}}-X_{u}^{\xi}\right|^{p}\right]}{\delta^{p}},
$$

with $\varrho=\vartheta^{\xi}$ in Case 1 and $\varrho=\widetilde{\vartheta}^{\xi}$ in Case 2.

(iii) For $\varepsilon>0, \epsilon \geq 0$, and $l=0, \ldots, N+1$, one has,

$$
\mathbb{E}\left[\left|\vartheta_{l}^{\xi, \varepsilon}-\vartheta_{l}^{\xi}\right|\right] \leq C_{\Lambda}^{\epsilon} \varepsilon^{1-\epsilon} .
$$

\subsubsection{Proof of Proposition 2.4}

Let in this section $\check{X}^{\xi_{n}}=X^{\xi_{n}}$, for $\xi_{n}=\left(t_{n}, x_{n}, k\right) \in \mathcal{E}$.

(i) When $t_{n} \downarrow t$, we want to control the difference between $\vartheta^{\xi}$ and $\vartheta^{\xi} \xi_{n}=\vartheta^{\xi_{n}}$ to prove the càglàd property. We shall use here the result of Case 1. First we know that

$$
\mathbb{E}\left[\sup _{u \in[0, T]}\left|X_{u}^{\xi_{n}}-X_{u}^{\xi}\right|^{p}\right] \leq C_{\Lambda}^{p}\left(\left|x-x_{n}\right|^{p}+\left|t-t_{n}\right|^{\frac{p}{2}}\right) .
$$

We then obtain, applying Lemma 5.1(ii), that

$$
\mathbb{E}\left[\left|\vartheta_{l}^{\xi}-\vartheta_{l}^{\xi_{n}}\right|\right] \leq\left|t-t_{n}\right|+C_{\Lambda}^{\epsilon} \delta_{n}^{1-\epsilon}+C_{\Lambda}^{p} \frac{\left|x-x_{n}\right|^{p}+\left|t-t_{n}\right|^{\frac{p}{2}}}{\delta_{n}^{p}}
$$

The proof is concluded by taking $\delta_{n}^{2}=\left|x-x_{n}\right| \vee\left|t-t_{n}\right|^{\frac{1}{2}}, p=2$ and letting $n$ go to $\infty$.

(ii) When $t_{n} \uparrow t$, we want to control the difference between $\widetilde{\vartheta}^{\xi}$ and $\vartheta^{\xi_{n}}$ to prove the làglàd ${ }^{7}$ property, assuming $x \notin \partial \mathcal{O}$. Since $x_{n} \rightarrow x$, we have for some $n \geq 0$ that $x_{n} \notin \partial \mathcal{O}$. We then argue as in (i), using this time the result of Case 2 in Lemma 5.1(i).

\subsubsection{Proof of Proposition 4.1}

Let in this section $\check{X}^{\xi^{\prime}}=\widehat{X}$, where $\xi^{\prime}:=(0, x, k)$, for $x \in \mathbb{R}^{q}, k \in \mathcal{K}$. We have here that $t=t^{\prime}=0$, so we are in Case 1 and basicly $\breve{\vartheta} \xi^{\prime}=\widehat{\vartheta}$. Applying Lemma 5.1(ii), we thus get, in view of (4.3),

$$
\mathbb{E}\left[\left|\vartheta_{l}-\widehat{\vartheta}_{l}\right|\right] \leq C_{\Lambda}^{\epsilon} \delta^{1-\epsilon}+C_{\Lambda}^{p} \frac{|\mathfrak{t}|^{\frac{p}{2}}}{\delta^{p}} .
$$

The proof is concluded by setting $\delta=|\mathfrak{t}|^{\frac{1}{2}-\frac{\theta}{2}}, \epsilon=\frac{\theta}{2}, p=\frac{1}{\theta}-2$, for $\theta$ and $|\mathfrak{t}|$ small enough.

\subsection{Proof of the BSDE results}

We denote by $\chi$ a positive random variable which may change from line to line but satisfies $\mathbb{E}\left[\chi^{p}\right] \leq C_{\Lambda}^{p}$, for $p \geq 1$.

\footnotetext{
${ }^{7}$ French acronym meaning "with left-hand and right-hand limits".
} 


\subsubsection{Proof of Proposition 4.6}

Let for $t \leq T$,

$$
\delta \widetilde{Y}_{t}=Y_{t}-\widetilde{\mathfrak{I}}_{t}^{\vartheta}, \delta Y_{t}=Y_{t-}-\mathfrak{I}_{t}^{\vartheta}, \delta Z_{t}=Z_{t}-\mathfrak{Z}_{t}^{\vartheta}, \delta f_{t}=f\left(t, X_{t}, Y_{t}\right)-f\left(t, X_{t}, \widetilde{\mathfrak{I}}_{t}^{\vartheta}\right) .
$$

Observe that is $\delta \widetilde{Y}$ continuous outside $\mathfrak{r}$ and that $\delta \widetilde{Y}_{t-}=\delta Y_{t}$ for $t \in(0, T]$, so that one has by (4.10), for $r \in \mathfrak{r}$,

$$
\left|\delta Y_{r}\right|=\left|Y_{r-}-\Im_{r}^{\vartheta}\right| \leq\left|\delta \tilde{Y}_{r}\right| .
$$

Applying Itô's formula to the càdlàg process $|\delta \widetilde{Y}|^{2}$ and observing that the local martingale term is in fact a martingale, we compute,

$$
\mathbb{E}_{r_{\imath}}\left[\left|\delta \widetilde{Y}_{t}\right|^{2}+\int_{t}^{r_{\imath+1}}\left|\delta Z_{u}\right|^{2} \mathrm{~d} u\right]=\mathbb{E}_{r_{\imath}}\left[\left|\delta \widetilde{Y}_{r_{\imath+1}-}\right|^{2}+2 \int_{t}^{r_{\imath+1}} \delta \widetilde{Y}_{s} \delta f_{s} \mathrm{~d} s+2 \int_{\left(t, r_{2+1}\right)} \delta \widetilde{Y}_{s} \mathrm{~d} A_{s}\right],
$$

for $t \in\left[r_{\imath}, r_{\imath+1}\right)$. Given (5.3), one thus gets by usual arguments, for $t \in\left[r_{\imath}, r_{\imath+1}\right)$,

$$
\mathbb{E}_{r_{\imath}}\left[\left|\delta \tilde{Y}_{t}\right|^{2}+\int_{t}^{r_{\imath+1}}\left|\delta Z_{s}\right|^{2} \mathrm{~d} s\right] \leq\left(1+C_{\Lambda}|\mathfrak{r}|\right) \mathbb{E}_{r_{2}}\left[\left|\delta \widetilde{Y}_{r_{\imath+1}}\right|^{2}+2 \int_{\left(t, r_{\imath+1}\right)} \delta \tilde{Y}_{s} \mathrm{~d} A_{s}^{+}-2 \int_{\left(t, r_{\imath+1}\right)} \delta \tilde{Y}_{s} \mathrm{~d} A_{s}^{-}\right] .
$$

We study the term related to the upper barrier. One has,

$$
\begin{aligned}
-\mathbb{E}_{r_{\imath}}\left[\int_{\left(t, r_{\imath+1}\right)} \delta \widetilde{Y}_{s} \mathrm{~d} A_{s}^{-}\right] & =\mathbb{E}_{r_{\imath}}\left[\int_{\left(t, r_{\imath+1}\right)}\left(\widetilde{\mathfrak{I}}_{s}^{\vartheta}-h\left(s, X_{s}\right)\right) \mathrm{d} A_{s}^{-}\right] \\
& =\mathbb{E}_{r_{\imath}}\left[\int_{\left(t, r_{\imath+1}\right)}\left(\widetilde{I}_{r_{\imath+1}}^{\vartheta}-h\left(s, X_{s}\right)\right) \mathrm{d} A_{s}^{-}+\int_{\left(t, r_{\imath+1}\right)} \int_{s}^{r_{\imath+1}} f\left(u, X_{u}, \widetilde{\mathfrak{I}}_{u}^{\vartheta}\right) \mathrm{d} u \mathrm{~d} A_{s}^{-}\right]
\end{aligned}
$$

where in particular the upper barrier minimality condition in $(\mathcal{S})$ was used in the first identity. The second term is bounded by

$$
\mathbb{E}_{r_{\imath}}\left[\chi|\mathfrak{r}|\left(A_{r_{\imath+1}-}^{-}-A_{r_{\imath}}^{-}\right)\right] \leq \mathbb{E}_{r_{\imath}}\left[\chi|\mathfrak{r}|\left(A_{r_{\imath+1}}^{-}-A_{r_{\imath}}^{-}\right)\right]
$$

since $f$ does not depend on $z$ and $A^{-}$is increasing. For the first term, we use the fact that $\mathrm{d} A^{-} \mathbf{1}_{\rrbracket \vartheta_{2 l}, \vartheta_{2 l+1} \llbracket}=0$, $0 \leq l \leq[(N+1) / 2]$, to obtain that

$$
\begin{aligned}
\mathbb{E}_{r_{\imath}}\left[\int_{\left(t, r_{\imath+1}\right)}\left(\Im_{r_{\imath+1}}^{\vartheta}-h\left(s, X_{s}\right)\right) \mathrm{d} A_{s}^{-}\right]= & \mathbb{E}_{r_{\imath}}\left[\sum_{l=1}^{[(N+1) / 2]} \int_{\left(t, r_{\imath+1}\right)}\left(\mathfrak{I}_{r_{\imath+1}}^{\vartheta}-h\left(s, X_{s}\right)\right) \mathbf{1}_{\left\{\vartheta_{2 l-1} \leq s \leq \vartheta_{2 l}\right\}} \mathrm{d} A_{s}^{-}\right] \\
& \leq \mathbb{E}_{r_{\imath}}\left[\sum_{l=1}^{[(N+1) / 2]} \int_{\left(t, r_{\imath+1}\right)}\left(h\left(r_{\imath+1}, X_{r_{\imath+1}}\right)-h\left(s, X_{s}\right)\right) \mathbf{1}_{\left\{\vartheta_{2 l-1} \leq s \leq \vartheta_{2 l}\right\}} \mathrm{d} A_{s}^{-}\right] \\
& \leq \mathbb{E}_{r_{\imath}}\left[\int_{\left(t, r_{\imath+1}\right)}\left(h\left(r_{\imath+1}, X_{r_{\imath+1}}\right)-h\left(s, X_{s}\right)\right) \mathrm{d} A_{s}^{-}\right]
\end{aligned}
$$

The proof is then concluded using the same argument as in the proof of Propositions 2.6.1 and 1.4.1 in [5]. 


\subsubsection{Proof of Proposition 4.7}

Let, for $t \leq T$,

$$
\begin{aligned}
& \delta \widetilde{\mathfrak{I}}_{t}=\widetilde{\mathfrak{I}}_{t}^{\vartheta}-\widetilde{\mathfrak{I}}_{t}^{\widehat{\vartheta}}, \delta \mathfrak{I}_{t}=\mathfrak{I}_{t}^{\vartheta}-\mathfrak{I}_{t}^{\widehat{\vartheta}}, \delta \mathfrak{Z}_{t}=\mathfrak{Z}_{t}^{\vartheta}-\mathfrak{Z}_{t}^{\widehat{\vartheta}} \\
& \eta_{t}=\left|\delta \widetilde{I}_{t}\right|^{2}-\left|\delta \widetilde{\mathfrak{I}}_{t}\right|^{2}, \delta f_{t}=f\left(t, X_{t}, \widetilde{\mathfrak{I}}_{t}^{\vartheta}\right)-f\left(t, X_{t}, \widetilde{\mathfrak{I}}_{t}^{\widehat{\vartheta}}\right) .
\end{aligned}
$$

Step 1. Applying Itô's formula to the càdlàg process $|\delta \widetilde{\mathfrak{I}}|^{2}$, we compute for $t \in\left[r_{\imath}, r_{\imath+1}\right)$

$$
\mathbb{E}_{r_{\imath}}\left[\left|\delta \widetilde{\mathfrak{I}}_{t}\right|^{2}+\int_{t}^{r_{\imath+1}}\left|\delta \mathfrak{Z}_{u}\right|^{2} \mathrm{~d} u\right]=\mathbb{E}_{r_{\imath}}\left[\left|\delta \widetilde{\mathfrak{I}}_{r_{\imath+1}}\right|^{2}+\eta_{r_{\imath+1}}+2 \int_{t}^{r_{\imath+1}} \delta \widetilde{\mathfrak{I}}_{s} \delta f_{s} \mathrm{~d} s\right]
$$

Usual arguments then yield that

$$
\sup _{s \in[t, T]} \mathbb{E}\left[\left|\delta \widetilde{\mathfrak{I}}_{s}\right|^{2}+\left|\delta \mathfrak{I}_{s}\right|^{2}+\int_{s}^{T}\left|\delta \mathfrak{Z}_{s}\right|^{2} \mathrm{~d} s\right] \leq C_{\Lambda} \mathbb{E}\left[\sum_{r \in \mathfrak{r}} \eta_{r}\right]
$$

recalling $\left|\delta \Im_{s}\right|^{2}=\eta_{s}+\left|\delta \widetilde{\mathfrak{I}}_{s}\right|^{2}$.

Step 2. In order to study the right-hand side term of (5.4), we introduce the processes defined by, for $r \in[0, T]$,

$$
\mathrm{I}_{r}=\sum_{l=1}^{[(N+1) / 2]} \mathbf{1}_{\left\{\vartheta_{2 l-1} \leq r \leq \vartheta_{2 l}\right\}}, \widehat{\mathrm{I}}_{r}=\sum_{l=1}^{[(N+1) / 2]} \mathbf{1}_{\left\{\widehat{\vartheta}_{2 l-1} \leq r \leq \widehat{\vartheta}_{2 l}\right\}},{ }^{c} \mathrm{I}_{r}=1-\mathrm{I}_{r},{ }^{c} \widehat{\mathrm{I}}_{r}=1-\widehat{\mathrm{I}}_{r} .
$$

Observe that $\mathrm{I}=1$ ( or $\widehat{\mathrm{I}}=1$ ) means that the upper barrier is activated for reflection.

$$
\begin{aligned}
\left|\delta \Im_{r}\right| & =\left|\mathcal{P}\left(r, X_{r}, \widetilde{\mathfrak{I}}_{r}^{\vartheta}\right)-\mathcal{P}\left(r, X_{r}, \widetilde{\mathfrak{I}}_{r}^{\vartheta}\right)\right| \\
& \leq\left|\delta \widetilde{\mathfrak{I}}_{r}\right|+\left[h\left(r, X_{r}\right)-\widetilde{\mathfrak{I}}_{r}^{\vartheta}\right]^{+} \mathrm{I}_{r}{ }^{c} \widehat{\mathrm{I}}_{r}+\left[h\left(r, X_{r}\right)-\widetilde{\mathfrak{I}}_{r} \widehat{\vartheta}^{+} \widehat{\mathrm{I}}_{r}{ }^{c} \mathrm{I}_{r} .\right.
\end{aligned}
$$

We thus compute, for $r \in \mathfrak{r}$,

$$
\eta_{r} \leq \mathbb{E}_{r}[\chi]\left(\left[h\left(r, X_{r}\right)-\widetilde{\mathfrak{I}}_{r}^{\vartheta}\right]^{+} \mathrm{I}_{r}{ }^{c} \widehat{\mathrm{I}}_{r}+\left[h\left(r, X_{r}\right)-\widetilde{\mathfrak{I}}_{r}^{\vartheta}\right]^{+} \widehat{\mathrm{I}}_{r}{ }^{c} \mathrm{I}_{r}\right)
$$

The two terms at the right-hand side of (5.8) are treated similarly, we thus concentrate on the first one.

Step 3. We have to take into account the fact that a reflection date may be a deactivation date for the upper boundary, i.e., for $r \in \mathfrak{r}$,

$$
\mathbb{E}_{r}[\chi]\left[\widetilde{\mathfrak{I}}_{r}^{\vartheta}-h\left(r, X_{r}\right)\right]^{+} \mathrm{I}_{r}{ }^{c} \widehat{\mathrm{I}}_{r}=\mathbb{E}_{r}[\chi]\left(\left[\widetilde{\mathfrak{I}}_{r}^{\vartheta}-h\left(r, X_{r}\right)\right]^{+}\right) \widehat{C}_{r}\left(\sum_{l=1}^{[(N+1) / 2]} \mathbf{1}_{\left\{r=\vartheta_{2 l}\right\}}+\sum_{l=1}^{[(N+1) / 2]} \mathbf{1}_{\left\{\vartheta_{2 l-1} \leq r<\vartheta_{2 l}\right\}}\right) .
$$

Step 3a. We study the first term in the right hand side of (5.9). We obviously have that $\mathbb{E}_{r}[\chi]\left[h\left(r, X_{r}\right)-\widetilde{\mathfrak{I}}_{r}^{\widehat{\vartheta}}-\right]^{+} \leq$ $\mathbb{E}_{r}[\chi]^{2}$, thus, since the $\vartheta_{l}$ are $\mathfrak{T}$-valued stopping-times,

$$
\sum_{r \in \mathfrak{r}} \mathbb{E}_{r}[\chi]\left[\widetilde{\mathfrak{I}}_{r}^{\hat{\vartheta}}-h\left(r, X_{r}\right)\right]^{+{ }^{c} \widehat{\mathrm{I}}_{r}} \sum_{l=1}^{[(N+1) / 2]} \mathbf{1}_{\left\{r=\vartheta_{2 l}\right\}} \leq \sum_{r \in \mathfrak{T}} \mathbb{E}_{r}[\chi]^{2}{\widehat{{ }^{c}}}_{r} \sum_{l=1}^{[(N+1) / 2]} \mathbf{1}_{\left\{r=\vartheta_{2 l}\right\}}
$$


Moreover, by definition of I and $\widehat{\mathrm{I}}$,

$$
\begin{aligned}
\sum_{r \in \mathfrak{T}} \mathbb{E}_{r}[\chi]^{2}{\widehat{\mathrm{I}_{r}}}_{r} \sum_{l=1}^{[(N+1) / 2]} \mathbf{1}_{\left\{r=\vartheta_{2 l}\right\}} & =\sum_{r \in \mathfrak{T}} \mathbb{E}_{r}[\chi]^{2} \widehat{c}_{r} \sum_{l=1}^{[(N+1) / 2]} \mathbf{1}_{\left\{r=\vartheta_{2 l}, r \neq \widehat{\vartheta}_{2 l}\right\}} \\
& \leq \sup _{r \in \mathfrak{T}} \mathbb{E}_{r}[\chi]^{2} \sum_{r \in \mathfrak{T}} \sum_{l=1}^{[(N+1) / 2]} \mathbf{1}_{\left\{\left|\vartheta_{2 l}-\widehat{\vartheta}_{2 l}\right| \geq|\mathfrak{T}| \sharp\right\}} .
\end{aligned}
$$

Using the Cauchy-Schwartz inequality with $\frac{1}{p}=1-\epsilon$, Doob's inequality and the Markov inequality, we obtain

$$
\mathbb{E}\left[\sum_{r \in \mathfrak{T}} \sum_{l=1}^{[(N+1) / 2]} \sup _{r \in \mathfrak{T}} \mathbb{E}_{r}[\chi]^{2} \mathbf{1}_{\left\{\left|\vartheta_{2 l}-\widehat{\vartheta}_{2 l}\right| \geq|\mathfrak{T}|^{\sharp} \mid\right\}}\right] \leq C_{\Lambda}^{\epsilon} \sum_{l=1}^{[(N+1) / 2]} \mathbb{E}\left[\left|\vartheta_{2 l}-\widehat{\vartheta}_{2 l}\right|\right]^{1-\epsilon} .
$$

Step 3b. We now study the last term in the right hand side of (5.9). On the event $\left\{\vartheta_{2 l-1} \leq r<\vartheta_{2 l}\right\}$, which is $\mathcal{F}_{r}$-measurable, the upper barrier is active on $\left[\vartheta_{2 l-1}, \vartheta_{2 l}\right]$, thus

$$
\widetilde{\mathfrak{I}}_{r}^{\vartheta}-h\left(r, X_{r}\right) \leq \mathbb{E}_{r}\left[h\left(r^{+}, X_{r^{+}}\right)-h\left(r, X_{r}\right)+\int_{r}^{r^{+}}\left|f\left(s, X_{s}, \Im_{s}^{\vartheta}\right)\right| \mathrm{d} s\right]
$$

where we set $r^{+}=\inf \{s \in \mathfrak{r} \mid s>r\} \wedge T$. One thus gets, using (Hb) or (Hb)',

$$
\left[\widetilde{\mathfrak{I}}_{r}^{\vartheta}-h\left(r, X_{r}\right)\right]^{+} \mathbf{1}_{\left\{\vartheta_{2 l-1} \leq r<\vartheta_{2 l}\right\}} \leq \mathbb{E}_{r}[\chi]|\mathfrak{r}|^{\alpha} .
$$

This leads to

$$
\sum_{r \in \mathfrak{r}}\left(\mathbb{E}_{r}[\chi]\left[\widetilde{\mathfrak{I}}_{r}^{\vartheta}-h\left(r, X_{r}\right)\right]^{+c} \widehat{\mathrm{I}}_{r} \sum_{l=1}^{[(N+1) / 2]} \mathbf{1}_{\left\{\vartheta_{2 l-1} \leq r<\vartheta_{2 l}\right\}}\right) \leq|\mathfrak{r}|^{\alpha} \mathbb{E}_{r}[\chi]^{2} \sum_{r \in \mathfrak{r}}\left({ }^{{ }^{c}} \widehat{\mathrm{I}}_{r} \sum_{l=1}^{[(N+1) / 2]} \mathbf{1}_{\left\{\vartheta_{2 l-1} \leq r<\vartheta_{2 l}\right\}}\right) .
$$

Moreover,

$$
\begin{aligned}
\sum_{r \in \mathfrak{r}} \sum_{l=1}^{[(N+1) / 2]}{ }^{{ }^{C}} \widehat{\mathrm{I}}_{r} \mathbf{1}_{\left\{\vartheta_{2 l-1} \leq r<\vartheta_{2 l}\right\}} & \leq \sum_{r \in \mathfrak{r}} \sum_{l=1}^{[(N+1) / 2]} \mathbf{1}_{\left\{\vartheta_{2 l-1} \leq r<\vartheta_{2 l}\right\}}\left(\mathbf{1}_{\left\{\widehat{\vartheta}_{2 l-1}>r\right\}}+\mathbf{1}_{\left\{r>\widehat{\vartheta}_{2 l}\right\}}\right) \\
& \leq \sum_{r \in \mathfrak{r}} \sum_{l=1}^{[(N+1) / 2]}\left(\mathbf{1}_{\left\{\left|\vartheta_{2 l}-\widehat{\vartheta}_{2 l}\right| \geq|\mathfrak{F}|^{\sharp}\right\}}+\mathbf{1}_{\left\{\left|\vartheta_{2 l-1}-\widehat{\vartheta}_{2 l-1}\right| \geq|\mathfrak{T}|^{\sharp}\right\}}\right) .
\end{aligned}
$$

We obtain combining the last inequality with (5.12) and using the Cauchy-Schwartz inequality with $\frac{1}{p}=1-\epsilon$, Doob's inequality and the Markov inequality

$$
\mathbb{E}\left[\sum_{r \in \mathfrak{r}}\left(\mathbb{E}_{r}[\chi]\left[\widetilde{\mathfrak{I}}_{r}^{\vartheta}-h\left(r, X_{r}\right)\right]^{+{ }^{c} \widehat{\mathrm{I}}_{r}} \sum_{l=1}^{[(N+1) / 2]} \mathbf{1}_{\left\{\vartheta_{2 l-1} \leq r<\vartheta_{2 l}\right\}}\right)\right] \leq|\mathfrak{r}|^{\alpha-1} C_{\Lambda}^{\epsilon} \sum_{l=1}^{[(N+1) / 2]} \mathbb{E}\left[\left|\vartheta_{2 l}-\widehat{\vartheta}_{2 l}\right|\right]^{1-\epsilon} .
$$

Step 4. The proof is concluded by combining (5.4) with (5.9), (5.10) and (5.12).

\subsubsection{Proof of Proposition 4.8}

Since

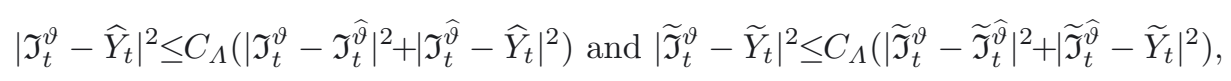


and

$$
\left|\mathfrak{Z}_{t}^{\vartheta}-\widehat{Z}_{t}\right|^{2} \leq C_{\Lambda}\left(\left|\mathfrak{Z}_{t}^{\vartheta}-\mathfrak{Z}_{t}^{\widehat{\vartheta}}\right|^{2}+\left|\mathfrak{Z}_{t}^{\widehat{\vartheta}}-\widehat{Z}_{t}\right|^{2}\right)
$$

it remains to study the error between $\left(\mathfrak{I}^{\widehat{\vartheta}}, \widetilde{\mathfrak{I}}^{\widehat{\vartheta}}, \mathfrak{Z}^{\widehat{\vartheta}}\right)$ and the continuous-time Euler scheme $(\widehat{Y}, \widetilde{Y}, \widehat{Z})$. We are thus going to show that

$$
\begin{array}{r}
\sup _{t \in[0, T]} \mathbb{E}\left[\left|\widetilde{\mathfrak{I}}_{t}^{\widehat{\vartheta}}-\widetilde{Y}_{t}\right|^{2}\right]+\sup _{t \in[0, T]} \mathbb{E}\left[\left|\widetilde{\mathfrak{I}}_{t}^{\widehat{\vartheta}}-\widehat{Y}_{t}\right|^{2}\right] \leq C_{\Lambda}|\mathfrak{t}| \\
\left\|\mathfrak{Z}^{\widehat{\vartheta}}-\widehat{Z}\right\|_{\mathcal{H}^{2}}^{2} \leq C_{\Lambda} \nu|\mathfrak{t}| .
\end{array}
$$

Step 1. We first prove (5.15). Toward this end, arguing as in the proof of Lemma 2.1 in [6] (See also Rem. 5.2 in [6]), one shows that under (Hb), for $t \in \mathfrak{t}$, there exists $S_{t}, Q_{t}$ in $\mathcal{F}_{t}$ such that $S_{t} \cap Q_{t}=\emptyset$ and

$$
\left|\widehat{I}_{t}^{\widehat{\vartheta}}-\widehat{Y}_{t}\right|^{2} \leq\left|\widetilde{\mathfrak{I}}_{t}^{\widehat{\vartheta}}-\widetilde{Y}_{t}\right|^{2} \mathbf{1}_{S_{t}}+C_{\Lambda}\left|X_{t}-\widehat{X}_{t}\right|^{2} \mathbf{1}_{Q_{t}}
$$

Observe in particular that for $t \notin \mathfrak{r}$, one can take $S_{t}=\Omega$ and $Q_{t}=\emptyset$ in (5.17) since, in this case, $\mathfrak{I}_{t}^{\widehat{\vartheta}}=\widetilde{\mathfrak{I}}_{t}^{\widehat{\vartheta}}$ and $\widehat{Y}_{t}=\widetilde{Y}_{t}$.

The proof of (5.15) is then similar to the proof of Proposition 5.1 (steps ia and ii) in [6]. Note that since $f$ does not depend on $z$ in the present case, the expression of $B_{i}$ in equation (5.5) of [6] reduces to

$$
B_{i}=\int_{t_{i-1}}^{t_{i}}\left(\left|X_{u}-\widehat{X}_{t_{i-1}}\right|^{2}+\left|\widetilde{\mathfrak{I}}_{u}^{\widehat{\vartheta}}-\widetilde{\mathfrak{I}}_{t_{i-1}}^{\widehat{\vartheta}}\right|^{2}\right) \mathrm{d} u .
$$

Observing that, for $u \in\left[t_{i-1}, t_{i}\right)$,

$$
\mathbb{E}\left[\left|\widetilde{\mathfrak{I}}_{u}^{\widehat{\vartheta}}-\widetilde{\mathfrak{I}}_{t_{i-1}}^{\widehat{\vartheta}}\right|^{2}\right] \leq C_{\Lambda} \mathbb{E}\left[\int_{t_{i-1}}^{t_{i}}\left|f\left(s, X_{s}, \widetilde{\mathfrak{I}}_{s}^{\widehat{\vartheta}}\right)\right|^{2} \mathrm{~d} s+\int_{t_{i-1}}^{t_{i}}\left|\mathfrak{Z}_{s}^{\widehat{\vartheta}}\right|^{2} \mathrm{~d} u\right]
$$

we obtain $\mathbb{E}\left[\sum_{i} B_{i}\right] \leq C_{\Lambda}|\mathfrak{t}|$. Inequalities (5.15) then follow from exactly the same arguments as in the proof of Proposition 5.1 of $[6]$.

Step 2. We now prove (5.16). Adapting to our context equation (5.10) in [6], we obtain

$$
\mathbb{E}\left[\int_{0}^{T}\left|\mathfrak{Z}_{t}^{\widehat{\vartheta}}-\widehat{Z}_{t}\right|^{2} \mathrm{~d} t\right] \leq C_{\Lambda}\left(\sup _{t \in \mathfrak{t}} \mathbb{E}\left[\left|\widetilde{I}_{t}^{\widehat{\vartheta}}-\widetilde{Y}_{t}\right|^{2}\right]+\mathbb{E}\left[\sum_{i} B_{i}\right]+\nu \mathbb{E}\left[\sup _{r \in \mathfrak{r}}\left|X_{r}-\widehat{X}_{r}\right|^{2}\right]\right)
$$

Combining the results of Step 1 with (4.3), we get (5.16).

Step 3. The proof of the Proposition is then concluded by combining (5.13)-(5.15) and (5.14)-(5.16) with Proposition 4.7.

\subsubsection{Proof of Theorems 4.3 and 4.4}

Proof of Theorem 4.3. Since

$$
\left|Y_{t-}-\widehat{Y}_{t}\right|^{2} \leq C_{\Lambda}\left(\left|Y_{t-}-\Im_{t}^{\vartheta}\right|^{2}+\left|\Im_{t}^{\vartheta}-\widehat{Y}_{t}\right|^{2}\right) \text { and }\left|Y_{t}-\widetilde{Y}_{t}\right|^{2} \leq C_{\Lambda}\left(\left|Y_{t}-\widetilde{\mathfrak{I}}_{t}^{\vartheta}\right|^{2}+\left|\widetilde{I}_{t}^{\vartheta}-\widetilde{Y}_{t}\right|^{2}\right)
$$

we obtain using Propositions 4.6 and 4.8 that

$$
\sup _{t \in[0, T]} \mathbb{E}\left[\left|Y_{t-}-\widehat{Y}_{t}\right|^{2}\right]+\sup _{t \in[0, T]} \mathbb{E}\left[\left|Y_{t}-\widetilde{Y}_{t}\right|^{2}\right] \leq C_{\Lambda}\left(|\mathfrak{t}|+|\mathfrak{r}|^{\alpha}+C_{\Lambda}^{\epsilon}|\mathfrak{r}|^{\alpha-1} \sum_{l=1}^{N} \mathbb{E}\left[\left|\widehat{\vartheta}_{l}-\vartheta_{l}\right|\right]^{1-\epsilon}\right) .
$$


Under $(\mathrm{Hb})^{\prime}$, the proof is concluded by using the last inequality together with Proposition 4.1 and letting $\mathfrak{r}=|\mathfrak{t}|$. Under (Hb), one chooses $|\mathfrak{r}| \sim|\mathfrak{t}|^{\frac{1}{2}}$.

Proof of Theorem 4.4. For $i<n$ and $t_{i} \in \mathfrak{t}$, let us define $\dot{Z}_{t_{i}}:=\mathbb{E}_{t_{i}}\left[\int_{t_{i}}^{t_{i+1}} Z_{s} \mathrm{~d} s\right] \mathbf{1}_{\left\{t_{i}<T\right\}}$ and then for $t \in[0, T] \backslash \mathfrak{t}$, $\dot{Z}_{t}=\dot{Z}_{\bar{t}}$. We observe that

$$
\mathbb{E}\left[\int_{0}^{T}\left|Z_{t}-\bar{Z}_{\bar{t}}\right|^{2} \mathrm{~d} t\right] \leq C_{\Lambda}\left(\mathbb{E}\left[\int_{0}^{T}\left|Z_{t}-\dot{Z}_{\bar{t}}\right|^{2} \mathrm{~d} t\right]+\mathbb{E}\left[\int_{0}^{T}\left|\dot{Z}_{\bar{t}}-\bar{Z}_{\bar{t}}\right|^{2} \mathrm{~d} t\right]\right)
$$

We then compute that

$$
\begin{aligned}
\mathbb{E}\left[\int_{0}^{T}\left|\dot{Z}_{\bar{t}}-\bar{Z}_{\bar{t}}\right|^{2} \mathrm{~d} t\right] & \leq C_{\Lambda} \mathbb{E}\left[\int_{0}^{T}\left|Z_{t}-\widehat{Z}_{t}\right|^{2} \mathrm{~d} t\right] \\
& \leq C_{\Lambda} \mathbb{E}\left[\int_{0}^{T}\left(\left|Z_{t}-\mathfrak{Z}_{t}^{\vartheta}\right|^{2}+\left|\mathfrak{Z}_{t}^{\vartheta}-\widehat{Z}_{t}\right|^{2}\right) \mathrm{d} t\right]
\end{aligned}
$$

Setting $|\mathfrak{r}| \sim|\mathfrak{t}|^{\frac{1}{2}}$, and combining Proposition 4.1, Proposition 4.6 and 4.8, we then have

$$
\mathbb{E}\left[\int_{0}^{T}\left|\dot{Z}_{\bar{t}}-\bar{Z}_{\bar{t}}\right|^{2} \mathrm{~d} t\right] \leq C_{\Lambda}^{\epsilon}|\mathfrak{t}|^{\frac{1}{4}-\epsilon}
$$

for $\epsilon>0$.

But we also have that

$$
\mathbb{E}\left[\int_{0}^{T}\left|Z_{t}-\dot{Z}_{\bar{t}}\right|^{2} \mathrm{~d} t\right] \rightarrow 0 \text { as }|\mathfrak{t}| \rightarrow 0
$$

Indeed $\dot{Z}$ is the best approximation of $Z$ in $L^{2}([0, T] \times \Omega)$ by processes wich are adapted and piecewise constant on $\mathfrak{t}$.

The proof of the Theorem is concluded by combining equations (5.19), (5.18) and (5.20).

\subsection{Proof of the PDE Results}

\subsubsection{Proof of Proposition 3.7}

Let $\mathcal{Y}^{\varepsilon_{n}, l, \xi}$ be the analog for the dilated domain $\mathcal{O}_{\varepsilon_{n}}$ of $\mathcal{Y}^{l, \xi}$ in Proposition 2.6, where the sequence $\varepsilon_{n}$ goes to 0 as $n \rightarrow \infty$.

In the same way as Lemma 5.1(ii) implies Proposition 2.4(i), one can imply from Lemma 5.1(iii) the existence of an extraction $\left(\varepsilon_{n^{\prime}}\right)_{n}$ for which, almost surely, $\vartheta^{\varepsilon_{n^{\prime}}, \xi}$ converges to $\vartheta^{\xi}$ as $n \rightarrow \infty$.

In the same way as Proposition 2.4(i) implies the result of the first bullet point in Proposition 2.6(ii) (see the related proof in [8]), one can in turn imply, from the almost sure convergence of $\vartheta^{\varepsilon_{n^{\prime}}, \xi}$ to $\vartheta^{\varepsilon, \xi}$, the convergence in $\mathcal{S}^{2} \times \mathcal{H}_{q}^{2} \times \mathcal{S}^{2}$ of $\mathcal{Y}^{\varepsilon_{n^{\prime}}, l, \xi}$ to $\mathcal{Y}^{l, \xi}$ as $n \rightarrow \infty$, for every $l=0, \ldots, N$. In view of Proposition 3.2 and identity (2.5) (both applied for $\mathcal{O}$ and $\mathcal{O}_{\varepsilon}$ ), one thus has, for $k \in K$,

$$
\left\{\begin{array}{l}
u_{\varepsilon_{n^{\prime}}, I}^{k}(t, x)=Y_{t}^{\varepsilon_{n^{\prime}}, 0, \xi} \rightarrow Y_{t}^{0, \xi}=u_{I}^{k}(t, x), k \in K \\
u_{\varepsilon_{n^{\prime}}, I}^{k}(t, x)=Y_{t}^{\varepsilon_{n^{\prime}}, 1, \xi} \rightarrow Y_{t}^{1, \xi}=u_{I}^{k}(t, x), k \notin K .
\end{array}\right.
$$

This proves (3.12).

Moreover the pointwise convergence (3.12) is uniform on every compact set of $\mathcal{E}_{I}^{*}$, by Dini's theorem applied to the functions $u_{I}$ and $u_{\varepsilon, I}$, which are continuous on $\mathcal{E}_{I}^{*}$. 


\subsubsection{Proof of Theorem 3.8}

First note that Theorem 3.8 can be reduced to the following three lemmas, which will be established below.

Lemma 5.2. $\bar{u}_{I}$, respectively $\underline{u}_{I}$, is upper, respectively lower semi-continuous on $\mathcal{E}_{I}$.

Since we already know that $\bar{u}_{I}$ is a continuous viscosity solution of (3.4) on $\mathcal{E}_{I}^{*}$, and given the definition of $\bar{u}_{I}$ at $T_{I}$, this implies in particular that $\bar{u}_{I}$ is a subsolution of (3.3)-(3.4) on $\mathcal{E}_{I}$.

Remark 5.3. One has likewise that $\bar{u}_{\varepsilon, I}$, respectively $\underline{u}_{\varepsilon, I}$, is a subsolution on $\mathcal{E}_{I}$, respectively supersolution on $\mathcal{E}_{I}$, of problem (3.14)-(3.15) (which is problem (3.3)-(3.4) with $\mathcal{O}$ replaced by $\mathcal{O}_{\varepsilon}$ ), for every $\varepsilon \geq 0$. Moreover, thanks to $(\mathrm{Hm})$, one also has that $\underline{u}_{\varepsilon, I}$ is a supersolution of $(3.3)-(3.4)$ (for the original domain $\mathcal{O}$ ) on $\mathcal{E}_{I}$.

Lemma 5.4. For every $\varepsilon>0, \underline{u}_{\varepsilon, I}$ is a strong supersolution of (3.3)-(3.4) on $\mathcal{E}_{I}$.

Given also Lemma 5.2, the comparison principle mentioned after Definition 3.4 then implies that $\mu \leq \lim \searrow_{\epsilon}{ } 0+$ $\underline{u}_{\varepsilon, I}$, for any subsolution $\mu$ of $(3.3)-(3.4)$ on $\mathcal{E}_{I}$. In particular, $\bar{u}_{I} \leq \lim \searrow_{\epsilon} \backslash 0+\underline{u}_{\varepsilon, I}$.

Lemma 5.5. $\lim \searrow_{\epsilon} 0+\underline{u}_{\varepsilon, I} \leq \bar{u}_{I}$.

Thus (3.13) is satisfied, $\mu \leq \bar{u}_{I}$ for every subsolution $\mu$ of (3.3)-(3.4) on $\mathcal{E}_{I}$, and Theorem 3.8 holds as a whole.

Next observe that Lemmas 5.2, 5.4 and 5.5 can in turn be reduced to showing that, at every $t=T_{I}$ :

- For Lemma 5.2: for every $x \in \partial \mathcal{O}$ and $k \in \mathcal{K}$,

$$
\check{u}_{I+1}^{k}(t, x) \geq \lim _{t_{n} \uparrow t-, x_{n} \rightarrow x} u_{I}^{k}\left(t_{n}, x_{n}\right), \text { respectively } \widehat{u}_{I+1}^{k}(t, x) \leq \lim _{t_{n} \uparrow t-, x_{n} \rightarrow x} u_{I}^{k}\left(t_{n}, x_{n}\right),
$$

where $\left(u_{I}^{k}\left(t_{n}, x_{n}\right)\right)_{n \geq 0}$ reaches $\limsup _{(t-, x)} u_{I}^{k} \quad:=\quad \lim \sup _{(s, y) \rightarrow(t, x) \text { with } s<t} u_{I}^{k}(s, y)$, respectively

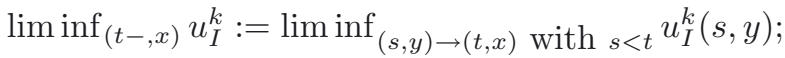

- For Lemma 5.4 (admitting Lem. 5.2, so $\underline{u}_{\varepsilon, I}$ supersolution of $(3.3)-(3.4)$ on $\mathcal{E}_{I}$ ):

$$
\widehat{u}_{\varepsilon, I+1} \geq \check{u}_{I+1}
$$

- For Lemma 5.5: for every $\left(t=T_{I}, x, k\right)$ with $x \in \partial \mathcal{O}$,

$$
\lim \searrow_{\epsilon} 0+\underline{u}_{\varepsilon, I}(t, x, k) \leq \bar{u}_{I}(t, x, k),
$$

where this "reduction" of Lemma 5.5 means that (5.23) only needs to be verified at the "critical" boundary points $\left(t=T_{I}, x, k\right)$ with $x \in \partial \mathcal{O}$ to which we reduce attention here, since it already holds at all the other points $(t, x, k)$ as

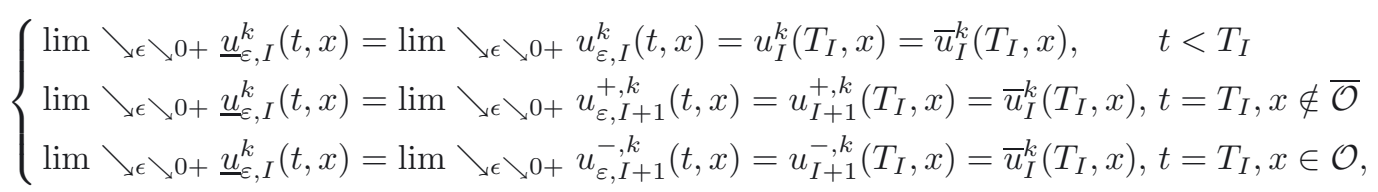

in which the middle identities result from Proposition 3.7.

Now, in order to establish (5.21), (5.22) and (5.23), there are five cases to consider, namely:

- $k \in K$ (easiest case),

- $k \notin K, \kappa_{I,+}^{k} \in K$ and $\kappa_{I,-}^{k} \notin K$, or the analogous but simpler case where $k \notin K$ and $\kappa_{I, \pm}^{k} \in K$,

- $k \notin K, \kappa_{I,+}^{k} \notin K$ and $\kappa_{I,-}^{k} \in K$, or the analogous but simpler case where $k \notin K$ and $\kappa_{I, \pm}^{k} \notin K$.

Moreover, the treatments of the "more difficult" cases $k \notin K, \kappa_{I,+}^{k} \in K$ and $\kappa_{I,-}^{k} \notin K$, or $k \notin K, \kappa_{I,+}^{k} \notin K$ and $\kappa_{I,-}^{k} \in K$, are symmetrical to each other. Considering the latter case, so in particular (cf. (3.5)):

$$
u_{\varepsilon, I+1}^{+, k}(t, x)=u_{\varepsilon}\left(t, x, \kappa_{I,+}^{k}\right), u_{\varepsilon, I+1}^{-, k}(t, x)=u_{\varepsilon}\left(t, x, \kappa_{I,-}^{k}\right) \wedge h(t, x),
$$

we now prove (5.21), (5.22) and (5.23) in this case, leaving the detail of the other cases to the reader. 
Proof of (5.21). We set $\xi_{n}=\left(t_{n}, x_{n}, k\right)$. In the considered case, since $k \notin K$, one has that $\vartheta_{0}^{\xi_{n}}=t_{n}=\vartheta_{1}^{\xi_{n}}<$ $\vartheta_{2}^{\xi_{n}}, u_{I}^{k}\left(t_{n}, x_{n}\right)=Y_{t_{n}}^{1, \xi_{n}}$, and:

- Whenever $X_{t}^{\xi_{n}} \notin \mathcal{O}$ :

$$
H_{t}^{\xi_{n}}=\kappa_{I,+}^{k} \notin K, \vartheta_{2}^{\xi_{n}}>t, u\left(t, \mathcal{X}_{t}^{\xi_{n}}\right)=Y_{t}^{1, \xi_{n}}
$$

- Whenever $X_{t}^{\xi_{n}} \in \mathcal{O}$ :

$$
H_{t}^{\xi_{n}}=\kappa_{I,-}^{k} \in K, \vartheta_{2}^{\xi_{n}}=t, u\left(t, \mathcal{X}_{t}^{\xi_{n}}\right)=Y_{t}^{2, \xi_{n}}, u\left(t, \mathcal{X}_{t}^{\xi_{n}}\right) \wedge h\left(t, X_{t}^{\xi_{n}}\right)=Y_{t}^{1, \xi_{n}},
$$

where the last identity results from (2.4).

Let us prove the left-hand-side inequality in (5.21) (leaving the other one to the reader), assuming that $\left(u_{I}^{k}\left(t_{n}, x_{n}\right)\right)_{n \geq 0}$ reaches $\lim \sup _{(t-, x)} u_{I}^{k}$. One has, recalling (5.25),

$$
\begin{aligned}
& \check{u}_{I+1}^{k}(t, x)-u_{I}^{k}\left(t_{n}, x_{n}\right)=\mathbb{E}\left\{\mathbf{1}_{X_{t}^{\xi_{n}} \notin \mathcal{O}}\left[\left(\check{u}_{I+1}^{k}(t, x)-u\left(t, X_{t}^{\xi_{n}}, \kappa_{I,+}^{k}\right)\right)+\left(Y_{t}^{1, \xi_{n}}-Y_{t_{n}}^{1, \xi_{n}}\right)\right]\right\} \\
& +\mathbb{E}\left\{\mathbf{1}_{X_{t}^{\xi_{n}} \in \mathcal{O}}\left[\left(\check{u}_{I+1}^{k}(t, x)-u\left(t, X_{t}^{\xi_{n}}, \kappa_{I,-}^{k}\right) \wedge h\left(t, X_{t}^{\xi_{n}}\right)\right)+\left(Y_{t}^{1, \xi_{n}}-Y_{t_{n}}^{1, \xi_{n}}\right)\right]\right\} \\
& \geq-\mathbb{E}\left|u\left(t, x, \kappa_{I,+}^{k}\right)-u\left(t, X_{t}^{\xi_{n}}, \kappa_{I,+}^{k}\right)\right| \\
& \quad-\mathbb{E}\left|u\left(t, x, \kappa_{I,-}^{k}\right) \wedge h\left(t, X_{t}^{\xi_{n}}\right)-u\left(t, X_{t}^{\xi_{n}}, \kappa_{I,-}^{k}\right) \wedge h\left(t, X_{t}^{\xi_{n}}\right)\right| \\
& \quad-\mathbb{E}\left|Y_{t}^{1, \xi_{n}}-Y_{t_{n}}^{1, \xi_{n}}\right|
\end{aligned}
$$

where, as $n \rightarrow \infty$ (cf. the proof of Prop. 3.2, see [8]):

- the first two terms go to 0 by continuity of the value function $u$ on $\mathcal{E}_{I+1}^{*}$, and

- the last term goes to 0 , by convergence of the $\mathcal{Y}^{\xi_{n}}$ (up to an extracted subsequence).

The left-hand-side inequality in (5.21) follows.

Proof of (5.22). One needs to prove

$$
\begin{cases}u_{\varepsilon}\left(t, x, \kappa_{I,+}^{k}\right) \geq u\left(t, x, \kappa_{I,+}^{k}\right), & x \notin \mathcal{O}_{\varepsilon} ; \\ u_{\varepsilon}\left(t, x, \kappa_{I,+}^{k}\right) \wedge u_{\varepsilon}\left(t, x, \kappa_{I,-}^{k}\right) \wedge h(t, x) \geq u\left(t, x, \kappa_{I,+}^{k}\right), & x \in \partial \mathcal{O}_{\varepsilon} ; \\ u_{\varepsilon}\left(t, x, \kappa_{I,-}^{k}\right) \wedge h(t, x) \geq u\left(t, x, \kappa_{I,+}^{k}\right), & x \in \mathcal{O}_{\varepsilon} \backslash \overline{\mathcal{O}} ; \\ u_{\varepsilon}\left(t, x, \kappa_{I,-}^{k}\right) \wedge h(t, x) \geq u\left(t, x, \kappa_{I,+}^{k}\right) \vee\left(u\left(t, x, \kappa_{I,-}^{k}\right) \wedge h(t, x)\right), & x \in \partial \mathcal{O} ; \\ u_{\varepsilon}\left(t, x, \kappa_{I,-}^{k}\right) \wedge h(t, x) \geq u\left(t, x, \kappa_{I,-}^{k}\right) \wedge h(t, x), & x \in \mathcal{O},\end{cases}
$$

which readily follows from $(\mathrm{Hm})$.

Proof of (5.23).

One has in the considered case $(c f .(5.25))$,

$$
\begin{aligned}
\underline{u}_{\varepsilon, I}^{k}\left(T_{I}, x\right) & =u_{\varepsilon}\left(T_{I}, x, \kappa_{I,+}^{k}\right)=u_{\varepsilon, I+1}\left(T_{I}, x, \kappa_{I,+}^{k}\right) \\
\bar{u}_{I}^{k}\left(T_{I}, x\right) & =u\left(T_{I}, x, \kappa_{I,+}^{k}\right) \vee\left(u\left(T_{I}, x, \kappa_{I,-}^{k}\right) \wedge h\left(T_{I}, x\right)\right) \\
& =u_{I+1}\left(T_{I}, x, \kappa_{I,+}^{k}\right) \vee\left(u_{I+1}\left(T_{I}, x, \kappa_{I,-}^{k}\right) \wedge h\left(T_{I}, x\right)\right)
\end{aligned}
$$

where $\lim \searrow \searrow 0+u_{\varepsilon, I+1}\left(t, x, \kappa_{I,+}^{k}\right)=u_{I+1}\left(t, x, \kappa_{I,+}^{k}\right)$, by application of Proposition 3.7. Hence (5.23) follows.

\subsubsection{Proof of Proposition 3.9}

This is obtained by a combination of classical viscosity arguments as in [2] (see also Subsect. 13.2.3 of [8]) and of arguments already used in the Proof of Theorem 3.8, so we shall only sketch the demonstration. 
(i) The middle inequality in (3.16) is immediate by definition of the envelopes of a scheme. Moreover, by classical viscosity solution arguments, $\underline{U}_{\varepsilon, I}$ and $\bar{U}_{\varepsilon, I}$ are respectively supersolutions and subsolutions of (3.14)-(3.15) on $\mathcal{E}_{I}$ (cf. Def. 3.4(ii) and (3.7)-(3.8) for the related definitions for $\varepsilon=0$ ). In particular, one has at $T_{I}$ :

$$
\underline{U}_{\varepsilon, I} \geq \widehat{u}_{\varepsilon, I+1}, \bar{U}_{\varepsilon, I} \leq \check{u}_{\varepsilon, I+1} .
$$

Moreover, by the dilation argument already used in the Proof of Proposition 3.8, one also has at $T_{I}$, for every $\varepsilon>0(c f .(5.22))$ :

$$
\widehat{u}_{\varepsilon, I+1} \geq \check{u}_{I+1}, \check{u}_{\varepsilon, I+1} \leq \widehat{u}_{2 \varepsilon, I+1} .
$$

In view also of Remark 5.3, one thus has that $\underline{U}_{\varepsilon, I}$ and $\bar{u}_{I}$ are respectively a strong supersolution and a subsolution on $\mathcal{E}_{I}$ of (3.3)-(3.4), whilst $\bar{U}_{\varepsilon, I}$ and $\underline{u}_{2 \varepsilon, I}$ are respectively a strong subsolution and a supersolution on $\mathcal{E}_{I}$ of (3.3)-(3.4) with $\mathcal{O}$ replaced by $\mathcal{O}_{2 \varepsilon}$, for every $\varepsilon>0$. The extreme inequalities in (3.16) thus follow by application of the comparison principle $(\mathrm{CP})$ to $\bar{u}_{I}$ and $\underline{U}_{\varepsilon, I}$ for the left side and $\bar{U}_{\varepsilon, I}$ and $\underline{u}_{2 \varepsilon, I}$ for the right one.

(ii) is then an elementary consequence of (3.16) joint to the fact that on $\mathcal{E}_{I}^{*}, \underline{u}_{2 \varepsilon, I}=u_{2 \varepsilon, I}$ converges locally uniformly to $u_{I}=\bar{u}_{I}$, by Proposition 3.7 .

\section{Appendix A. Proof of Lemma 5.1}

\section{A.1. Proof of Part (i)}

We only consider the case of $t=0$, the arguments for general time initial condition being exactly the same.

Using Itô Formula, we compute

$$
\mathrm{d}\left(X_{s}\right)=\mathrm{d}\left(X_{0}\right)+\int_{0}^{s} \mathcal{G} d\left(u, X_{u}\right) \mathrm{d} u+\int_{0}^{s} \partial_{x} \mathrm{~d} \sigma\left(u, X_{u}\right) \mathrm{d} W_{u} .
$$

We define a new probability $\mathbb{Q} \sim \mathbb{P}$ whose density is given by

$$
\mathrm{e}^{-\int_{0}^{T} \lambda_{u} \mathrm{~d} W_{u}-\frac{1}{2} \int_{0}^{T}\left|\lambda_{u}\right|^{2} \mathrm{~d} u} \text { where } \lambda_{u}:=\left(\left(\partial_{x} d \sigma\right)^{\top}\left(\partial_{x} d a \partial_{x} d^{\top}\right)^{-1} \mathcal{G} d\right)\left(u, X_{u}\right)
$$

and the process $W^{\mathbb{Q}}$ by $\mathrm{d} W_{u}^{\mathbb{Q}}:=\mathrm{d} W_{u}+\lambda_{u} \mathrm{~d} u$. Observe that Novikov's condition holds since $\lambda$ is bounded, under $(\mathrm{Hx})-(\mathrm{Ho})$. Thus, it follows from Girsanov's Theorem that $W^{\mathbb{Q}}$ is a brownian motion under $\mathbb{Q}$. Equation (A.1) reads then

$$
\mathrm{d}\left(X_{s}\right)=\mathrm{d}\left(X_{s}\right)+\int_{0}^{s} \partial_{x} d \sigma\left(u, X_{u}\right) \mathrm{d} W_{u}^{\mathbb{Q}} .
$$

For $T_{I} \in \mathfrak{T} \backslash\{0\}$, using Holder's inequality, one computes that

$$
\mathbb{P}\left\{\left|\mathrm{d}\left(X_{T_{I}}\right)\right| \leq \delta\right\} \leq C_{\Lambda}^{\epsilon} \mathbb{Q}\left\{\left|\mathrm{d}\left(X_{T_{I}}\right)\right| \leq \delta\right\}^{1-\epsilon} .
$$

The proof is then concluded using Corollary 2.1.1 in [23] and working under $\mathbb{Q}$.

\section{A.2. Proof of Part (ii)}

We first define for $\delta>0$, the sets

$$
\widehat{\Omega}^{\delta}=\left\{\sup _{u \in[0, T]}\left|\check{X}_{u}^{\xi^{\prime}}-X_{u}^{\xi}\right|<\delta\right\},{ }^{c} \widehat{\Omega}^{\delta}=\Omega \backslash \widehat{\Omega}^{\delta} .
$$

We consider the two different cases. 
Case 1. (a) By definition of $\vartheta^{\xi}, \check{\vartheta}^{\xi^{\prime}}$, we have that $\mathbb{E}\left[\left|\vartheta_{0}^{\xi}-\breve{\vartheta}_{0}^{\xi^{\prime}}\right|\right]=\left|t-t^{\prime}\right|$, and obviously, for $l \geq 1$,

$$
\mathbb{E}\left[\left|\vartheta_{l}^{\xi}-\check{\vartheta}_{l}^{\xi^{\prime}}\right|\right]=\mathbb{E}\left[\left|\vartheta_{l}^{\xi}-\check{\vartheta}_{l}^{\xi^{\prime}}\right| \mathbf{1}_{c \widehat{\Omega}^{\delta}}\right]+\mathbb{E}\left[\left|\vartheta_{l}^{\xi}-\check{\vartheta}_{l}^{\xi^{\prime}}\right| \mathbf{1}_{\widehat{\Omega}^{\delta} \cap\left\{\vartheta_{l}^{\xi} \neq \check{\vartheta}_{l}^{\xi^{\prime}}\right\}}\right]
$$

Markov's inequality applied on ${ }^{c} \widehat{\Omega}^{\delta}$, and the bound $\left|\vartheta_{l}^{\xi}-\breve{\vartheta}_{l}^{\xi}\right| \leq T$, yield that

$$
\mathbb{E}\left[\left|\vartheta_{l}^{\xi}-\check{\vartheta}_{l}^{\xi^{\prime}}\right| \mathbf{1}_{c \widehat{\Omega}^{\delta}}\right] \leq C_{\Lambda}^{p} \frac{\mathbb{E}\left[\sup _{u \in\left[\mathcal{T}^{t}, T\right]}\left|\check{X}_{u}^{\xi^{\prime}}-X_{u}^{\xi}\right|^{p}\right]}{\delta^{p}}
$$

for $p_{i} 0$.

(b) We now work on the second term of the right-hand side of (A.3). By definition of $\vartheta^{\xi}, \check{\vartheta}^{\xi^{\prime}}$, if $k \notin K$, we have $\mathbb{E}\left[\left|\vartheta_{1}^{\xi}-\breve{\vartheta}_{1}^{\xi^{\prime}}\right| \mathbf{1}_{\{k \notin K\}}\right]=\left|t-t^{\prime}\right|$. We are going to prove a control between $\vartheta^{\xi}$ and $\check{\vartheta}^{\xi}$, for $l \geq 2$, and for $l=1$, $k \in K$. To this end, we observe that

$$
\mathbf{1}_{\left\{X_{T_{I}}^{\xi} \in \mathcal{O}\right\}}=\mathbf{1}_{\left\{\check{X}_{T_{I}}^{\xi^{\prime}} \in \mathcal{O}\right\}}, \forall T_{I} \in \mathfrak{T}^{t} \Longrightarrow H^{\xi}=\check{H}^{\xi^{\prime}}
$$

thus for $l \geq 2, \vartheta_{l}^{\xi}=\check{\vartheta}_{l}^{\xi^{\prime}}$ and if $k \in K, \vartheta_{1}^{\xi}=\check{\vartheta}_{1}^{\xi^{\prime}}$.

We then introduce the set

$$
\Omega_{1}=\bigcup_{T_{I} \in \mathfrak{T}^{t}}\left(\left\{\mathrm{~d}\left(X_{T_{I}}^{\xi}\right) \geq 0\right\} \cap\left\{\mathrm{d}\left(\check{X}_{T_{I}}^{\xi^{\prime}}\right)<0\right\}\right) \cup\left(\left\{\mathrm{d}\left(X_{T_{I}}^{\xi}\right)<0\right\} \cap\left\{\mathrm{d}\left(\check{X}_{T_{I}}^{\xi^{\prime}}\right) \geq 0\right\}\right) .
$$

Since $d$ is 1-Lipschitz continuous, by definition of $\widehat{\Omega}^{\delta}$, we have

$$
\widehat{\Omega}^{\delta} \cap \Omega_{1} \subset \bigcup_{T_{I} \in \mathfrak{T}^{t}}\left\{\left|\mathrm{~d}\left(X_{T_{I}}^{\xi}\right)\right| \leq \delta\right\}=: \bar{\Omega}
$$

Using (A.5), we have that, for $l \geq 2,\left\{\vartheta_{l}^{\xi} \neq \breve{\vartheta}_{l}^{\xi^{\prime}}\right\} \subset \Omega_{1}$ and if $k \in K,\left\{\vartheta_{1}^{\xi} \neq \check{\vartheta}_{1}^{\xi^{\prime}}\right\} \subset \Omega_{1}$. Thus, for $l \geq 2$, $\widehat{\Omega}^{\delta} \cap\left\{\vartheta_{l}^{\xi} \neq \breve{\vartheta}_{l}^{\xi^{\prime}}\right\} \subset \bar{\Omega}$ and if $k \in K, \widehat{\Omega}^{\delta} \cap\left\{\vartheta_{1}^{\xi} \neq \breve{\vartheta}_{1}^{\xi^{\prime}}\right\} \subset \bar{\Omega}$.

Using the result of Part (i), one then gets,

$$
\mathbb{E}\left[\left|\vartheta_{l}^{\xi}-\check{\vartheta}_{l}^{\xi^{\prime}}\right| \mathbf{1}_{\widehat{\Omega}^{\delta} \cap\left\{\vartheta_{l}^{\xi} \neq \check{\vartheta}_{l}^{\xi^{\prime}}\right\}}\right] \leq C_{\Lambda} \delta^{1-\epsilon},
$$

for $l \geq 2$ and $l=1$, if $k \in K$. In this case, the proof is concluded combining the last inequality with (A.4) and (A.3).

Case 2. In this case, $\mathfrak{T}^{t^{\prime}}=\mathfrak{T}^{t} \cup\{t\}$. As in Case 1 (a) above, we compute

$$
\mathbb{E}\left[\left|\widetilde{\vartheta}_{l}^{\xi}-\check{\vartheta}_{l}^{\xi^{\prime}}\right|\right] \leq C_{\Lambda}^{p} \frac{\mathbb{E}\left[\sup _{u \in\left[\mathcal{T}^{t}, T\right]}\left|\check{X}_{u}^{\xi^{\prime}}-X_{u}^{\xi}\right|^{p}\right]}{\delta^{p}}+\mathbb{E}\left[\left|\widetilde{\vartheta}_{l}^{\xi}-\check{\vartheta}_{l}^{\xi^{\prime}}\right| \mathbf{1}_{\widehat{\Omega}^{\delta} \cap\left\{\widetilde{\vartheta}_{l}^{\xi} \neq \check{\vartheta}_{l}^{\xi^{\prime}}\right\}}\right],
$$

for $l \geq 0$ and $p, \epsilon i$. Recall that by definition of $\widetilde{\vartheta} \xi, \check{\vartheta}^{\prime}, \mathbb{E}\left[\left|\widetilde{\vartheta}_{0}^{\xi}-\check{\vartheta}_{0}^{\xi^{\prime}}\right|\right]=\left|t-t^{\prime}\right|$ and if $k \notin K, \mathbb{E}\left[\left|\widetilde{\vartheta}_{1}^{\xi}-\check{\vartheta}_{1}^{\xi^{\prime}}\right|\right]=$ $\left|t-t^{\prime}\right|$. Regarding the last term of (A.6), we observe here that

$$
\mathbf{1}_{\left\{X_{T_{I}}^{\xi} \in \mathcal{O}\right\}}=\mathbf{1}_{\left\{\check{X}_{T_{I}}^{\xi^{\prime}} \in \mathcal{O}\right\}}, \forall T_{I} \in \mathfrak{T}^{t} \cup\{t\} \Longrightarrow \widetilde{H}^{\xi}=\check{H}^{\xi^{\prime}} .
$$


The set $\Omega_{1}$ is now replaced by

$$
\Omega_{2}=\bigcup_{T_{I} \in \mathfrak{T}^{t} \cup\{t\}}\left(\left\{\mathrm{d}\left(X_{T_{I}}^{\xi}\right) \geq 0\right\} \cap\left\{\mathrm{d}\left(\check{X}_{T_{I}}^{\xi^{\prime}}\right)<0\right\}\right) \cup\left(\left\{\mathrm{d}\left(X_{T_{I}}^{\xi}\right)<0\right\} \cap\left\{\mathrm{d}\left(\check{X}_{T_{I}}^{\xi^{\prime}}\right) \geq 0\right\}\right)
$$

The difference with Case $1(\mathrm{~b})$ is that the reunion is on $\mathfrak{T}^{t} \cup\{t\}$. But, since for $\delta$ small enough $\left\{\left|\mathrm{d}\left(X_{t}^{\xi}\right)\right|<\delta\right\}=\emptyset$, we have

$$
\widehat{\Omega}^{\delta} \cap \Omega_{2} \subset \bar{\Omega} .
$$

The proof is then concluded arguing as in Case 1(b).

\section{A.3. Proof of Part (iii)}

As in Part (ii), we observe that

$$
\mathbf{1}_{\left\{X_{T_{I}}^{\xi} \in \mathcal{O}\right\}}=\mathbf{1}_{\left\{X_{T_{I}}^{\xi} \in \mathcal{O}_{\varepsilon}\right\}}, \forall T_{I} \in \mathfrak{T}^{t} \Longrightarrow H^{\xi}=H^{\xi, \varepsilon},
$$

thus for $l \geq 0, \vartheta_{l}^{\xi, \varepsilon}=\vartheta_{l}^{\xi}$.

We then introduce the set

$$
\Omega_{3}=\bigcup_{T_{I} \in \mathfrak{T}^{t}}\left(\left\{\mathrm{~d}\left(X_{T_{I}}^{\xi}\right) \geq 0\right\} \cap\left\{\mathrm{d}_{\varepsilon}\left(X_{T_{I}}^{\xi}\right)<0\right\}\right) \cup\left(\left\{\mathrm{d}\left(X_{T_{I}}^{\xi}\right)<0\right\} \cap\left\{\mathrm{d}_{\varepsilon}\left(X_{T_{I}}^{\xi}\right) \geq 0\right\}\right) .
$$

where $d_{\varepsilon}$ is the distance function associated to $\mathcal{O}_{\varepsilon}$. By definition of $\mathcal{O}_{\varepsilon}$, we have

$$
\Omega_{3} \subset \bigcup_{T_{I} \in \mathfrak{T}^{t}}\left\{\left|\mathrm{~d}\left(X_{T_{I}}^{\xi}\right)\right| \leq \varepsilon\right\}
$$

The proof is then concluded using Part (i).

\section{REFERENCES}

[1] V. Bally and G. Pagès, Error analysis of the quantization algorithm for obstacle problems. Stoch. Process. Appl. 106 (2003) $1-40$.

[2] G. Barles and P.E. Souganidis, Convergence of approximation schemes for fully nonlinear second order equations. Asymptot. Anal. 4 (1991) 271-283.

[3] B. Bouchard and J.-F. Chassagneux, Discrete time approximation for continuously and discretely reflected BSDEs. Stoch. Process. Appl. 118 (2008) 2269-2293.

[4] B. Bouchard and S. Menozzi, Strong Approximations of BSDEs in a domain. Bernoulli 15 (2009) 1117-1147.

[5] J.-F. Chassagneux, Processus réfléchis en finance et probabilité numérique. Ph.D. thesis Université Paris Diderot - Paris (2008) 7.

[6] J.-F. Chassagneux, Discrete time approximation of doubly reflected BSDEs. Adv. Appl. Probab. 41 (2009) 101-130.

[7] M. Crandall, H. Ishii and P.-L. Lions, User's guide to viscosity solutions of second order partial differential equations. Bull. Amer. Math. Soc. (1992).

[8] S. Crépey, Financial Modeling: A Backward Stochastic Differential Equations Perspective. Springer Finance Textbooks. Springer (2013).

[9] S. Crépey and A. Matoussi, Reflected and doubly reflected BSDEs with jumps: A priori estimates and comparison principle. Ann. Appl. Probab. 18 (2008) 2041-2069.

[10] S. Crépey and A. Rahal, Pricing Convertible Bonds with Call Protection. J. Comput. Finance 15 (2011/12) 37-75.

[11] J. Cvitanić and I. Karatzas, Backward stochastic differential equations with reflection and Dynkin games. Ann. Probab. 24 (1996) 2024-2056.

[12] E.B. Dynkin, Game variant of a problem on optimal stopping. Soviet Math. Dokl. 10 (1969) 270-274.

[13] N. El Karoui, E. Kapoudjian, C. Pardoux and S. Peng, and M.-C. Quenez, Reflected solutions of backward SDE's, and related obstacle problems for PDE's. Ann. Probab. 25 (1997) 702-737.

[14] N. El Karoui, S. Peng and M.-C. Quenez, Backward stochastic differential equations in finance. Math. Finance 7 (1997) 1-71. 
[15] E. Gobet and A. Makhlouf L2-time regularity of BSDEs with irregular terminal functions. Stoch. Process. Appl. 120 (2010) $1105-1132$.

[16] S. Hamadène, Reflected BSDEs with Discontinuous Barrier and Application. Stoch. Stoch. Reports 74 (2002) 571-596.

[17] S. Hamadène and M. Hassani, BSDEs with two reflecting barriers driven by a Brownian motion and an independent Poisson noise and related Dynkin game. Electr. J. Probab. 11 (2006) 121-145.

[18] S. Hamadène and M. Hassani, BSDEs with two reflecting barriers: the general result. Probab. Theory Relat. Fields 132 (2005) $237-264$.

[19] S. Hamadène, M. Hassani and Y. Ouknine, BSDEs with general discontinuous reflecting barriers without Mokobodski's condition. Bull. Sci. Math. 134 (2010) 874-899.

[20] Y. Kifer, Game options. Fin. Stoch. 4 (2000) 443-463.

[21] P.E. Kloeden and E. Platen, Numerical Solution of Stochastic Differential Equations. Springer (2000).

[22] J.-P. Lepeltier and M. Xu, Reflected backward stochastic differential equations with two RCLL barriers. ESAIM: PS 4 (2007) $3-22$.

[23] D. Nualart, The Malliavin Calculus and Related Topics, 2nd Edition. Springer (2006). 